\title{
Local rigidity of group actions: past, present, future
}

\author{
DAVID FISHER
}

To Anatole Katok on the occasion of his 60th birthday.

\begin{abstract}
This survey aims to cover the motivation for and history of the study of local rigidity of group actions. There is a particularly detailed discussion of recent results, including outlines of some proofs. The article ends with a large number of conjectures and open questions and aims to point to interesting directions for future research.
\end{abstract}

\section{Prologue}

Let $\Gamma$ be a finitely generated group, $D$ a topological group, and $\pi: \Gamma \rightarrow D$ a homomorphism. We wish to study the space of deformations or perturbations of $\pi$. Certain trivial perturbations are always possible as soon as $D$ is not discrete, namely we can take $d \pi d^{-1}$ where $d$ is a small element of $D$. This motivates the following definition:

DEFINITION 1.1. Given a homomorphism $\pi: \Gamma \rightarrow D$, we say $\pi$ is locally rigid if any other homomorphism $\pi^{\prime}$ which is close to $\pi$ is conjugate to $\pi$ by a small element of $D$.

We topologize $\operatorname{Hom}(\Gamma, D)$ with the compact open topology which means that two homomorphisms are close if and only if they are close on a generating set for $\Gamma$. If $D$ is path connected, then we can define deformation rigidity instead, meaning that any continuous path of representations $\pi_{t}$ starting at $\pi$ is conjugate to the trivial path $\pi_{t}=\pi$ by a continuous path $d_{t}$ in $D$ with $d_{0}$ being

Author partially supported by NSF grant DMS-0226121 and a PSC-CUNY grant. 
the identity in $D$. If $D$ is an algebraic group over $\mathbb{R}$ or $\mathbb{C}$, it is possible to prove that deformation rigidity and local rigidity are equivalent since $\operatorname{Hom}(\Gamma, D)$ is an algebraic variety and the action of $D$ by conjugation is algebraic; see [Mu], for example. For $D$ infinite dimensional and path-connected, this equivalence is no longer clear.

The study of local rigidity of lattices in semisimple Lie groups is probably the beginning of the general study of rigidity in geometry and dynamics, a subject that is by now far too large for a single survey. See [Sp1] for the last attempt at a comprehensive survey and [Sp2] for a more narrowly focused updating of that survey. Here we abuse language slightly by saying a subgroup is locally rigid if the defining embedding is locally rigid as a homomorphism. See subsection 3.1 for a brief history of local rigidity of lattices and some discussion of subsequent developments that are of particular interest in the study of rigidity of group actions.

In this article we will focus on a survey of local rigidity when $D=\operatorname{Diff}^{\infty}(M)$ or occasionally $D=\operatorname{Diff}^{k}(M)$ for some finite $k$. Here we often refer to a homomorphism $\pi: \Gamma \rightarrow \operatorname{Diff}^{\infty}(M)$ as an action, since it can clearly be thought of as $C^{\infty}$ action $\pi: \Gamma \times M \rightarrow M$. We will consistently use the same letter $\pi$ to denote either the action or the homomorphism to $\operatorname{Diff}^{\infty}(M)$. The title of this article refers to this interpretation of $\pi$ as defining a group action. In this context, one considers rigidity of actions of connected groups as well as of discrete groups. In cases where $\Gamma$ has any topology, we will always only study continuous actions, i.e. ones for which the defining homomorphism $\pi$ is a continuous map.

One can, in this context, develop more refined notions of local rigidity, since the topology on $\operatorname{Diff}^{\infty}(M)$ is an inverse limit topology. This means that two $C^{\infty}$ diffeomorphisms of $M$ are close precisely when they are $C^{k}$ close for some large value of $k$. The most exhaustive definition of local rigidity is probably the following:

DEFINITION 1.2. Let $\Gamma$ be a discrete group and $\pi: \Gamma \rightarrow \operatorname{Diff}^{k}(M)$ a homomorphism where $k$ is either a positive integer or $\infty$. We say that $\pi$ is $C^{k, l, i, j, m}$ locally rigid if any $\pi^{\prime}: \Gamma \rightarrow \operatorname{Diff}^{l}(M)$ which is close to $\pi$ in the $C^{i}$ topology is conjugate to $\pi$ by a $C^{j}$ diffeomorphism $\phi$ which is $C^{m}$ small. Here $l, i, j, m$ are all either nonnegative integers or $\infty$ and the only a priori constraint are $i \leq \min (k, l)$ and $m \leq j$. When $j=0$, we will call the action stable or structurally stable. When $j>0$, we will call the action locally rigid or simply rigid.

We will avoid using this cumbersome notation when at all possible. There is a classical, dynamical notion of structural stability which is equivalent to $C^{1,1,1,0,0}$ local rigidity. I.e. a $C^{1}$ action $\pi$ of a group $\Gamma$ is structurally stable if any $C^{1}$ close $C^{1}$ action of $\Gamma$ is conjugate to $\pi$ by a small homeomorphism. For 
actions of $\mathbb{Z}$ this notion arose in hyperbolic dynamics in the work of Anosov and Smale [An; Sm]. From a dynamical point of view structural stability is important since it allows one to control dynamical properties of an open set of actions in $\operatorname{Diff}^{1}(M)$. Local rigidity can be viewed as a strengthening of this property in that it shows that an open set of actions is exhausted by smooth conjugates of a single action.

Though actions of $\mathbb{Z}$ and free groups on $k$ generators are often structurally stable, they are never locally rigid, and it is an interesting question as to how "large" a group needs to be in order to have actions which are locally rigid. Many of the original questions and theorems concerning local rigidity were for lattices in higher rank semisimple Lie groups, where here higher rank means that all simple factors have real rank at least 2. (See subsection 2.1 for a definition of rank.) Fairly early in the theory it became clear that local rigidity often held, and was in fact easier to prove, for certain actions of higher rank abelian groups, i.e. $\mathbb{Z}^{k}$ for $k \geq 2$, see [KL1]. In addition, local rigidity results have been proven for actions of a wider variety of groups, including

(1) certain non-volume-preserving actions of lattices in $S O(1, n)$ in [Kan1]

(2) all isometric actions of groups with property $(T)$ in [FM2],

(3) certain affine actions of lattices in $\operatorname{SP}(1, n)$ in [Hi].

There is extremely interesting related work of Ghys, older than the work just mentioned, which shows that the space of deformations of certain actions of surface groups on the circle is finite dimensional [Gh1; Gh2; Gh3]. Ghys also proved some very early results on local and global rigidity of very particular actions of connected solvable groups, see [GhS; Gh1] and subsection 4.2.

The study of local rigidity of group actions has had three primary historical motivations, one from the theory of lattices in Lie groups, one from dynamical systems theory and a third from the theory of foliations. (This statement is perhaps a bit coarse, and there is heavy overlap between these motivations, particularly the second and the third.) The first is the general study of rigidity of actions of large groups, as discussed in [Z3; Z4], see [La; FK] for more up to date surveys. This area is motivated by the study of rigidity of lattices in semisimple Lie groups, particularly by Margulis' superrigidity theorem and it's nonlinear generalization by Zimmer to a cocycle superrigidity theorem, see subsection 3.1 and [Z4] for more discussion. This motivation also stems from an analogy between semisimple Lie groups and diffeomorphism groups. When $M$ is a compact manifold, not only is $\operatorname{Diff}^{\infty}(M)$ an infinite dimensional Lie group, but its connected component is simple. Simplicity of the connected component of $\operatorname{Diff}^{\infty}(M)$ was proven by Thurston using results of Epstein and Herman [Th2; Ep; Hr]. Herman had used Epstein's work to see that the connected component of Diff ${ }^{\infty}\left(\mathbb{T}^{n}\right)$ is simple and Thurston's proof of the general case uses this. See 
also further work on the topic by Banyaga and Mather [Ba1; Mt1; Mt2; Mt3], as well as Banyaga's book [Ba2].

The dynamical motivation for studying rigidity of group actions comes from the study of structural stability of diffeomorphisms and flows in hyperbolic dynamics, see the introduction of [KS2]. This area provides many of the basic techniques by which results in the area have been proven, particularly early in the history of the field. Philosophically, hyperbolic diffeomorphisms are structurally stable, group actions generated by structurally stable diffeomorphisms are quite often structurally stable, and the presence of a large group action frequently allows one to improve the regularity of the conjugacy. See subsection 3.2 for a brief history of relevant results on structural stability and subsections 4.1, 4.2, and 5.1 for some applications of these ideas.

The third motivation for studying rigidity of group actions comes from the theory of foliations. Many techniques and ideas in this area are also related to work on hyperbolic dynamics, and many of the foliations of interest are dynamical foliations of hyperbolic dynamical systems. A primary impetus in this area is the theory of codimension one foliations, and so many of the ideas here were first developed either for groups acting on the circle or for actions of connected groups on manifolds only one dimension larger then the acting group. See particularly [GhS; Gh1] for the early history of these developments.

Some remarks on biases and omissions. Like any survey of this kind, this work is informed by it's authors biases and experiences. The most obvious of these is that my point of view is primarily motivated by the study of rigidity properties of semisimple Lie groups and their lattices, rather than primarily motivated by hyperbolic dynamics or foliation theory. This informs the biases of this article and a very different article would result from different biases.

There are two large omissions in this article. The first omission is that it is primarily occupied with local rigidity of discrete group actions. When similar results are known for actions of Lie groups, they are mentioned, though frequently only special cases are stated. This is partially because results in this context are often complicated by the need to consider time changes, and I did not want to dwell on that issue here. The second omission is that little to no care is taken to state optimal results relating the various constants in $C^{k, l, i, j, m}$ local rigidity. Dwelling on issues of regularity seemed likely to obscure the main line of the developments, so many results are stated without any explicit mention of regularity. Usually this is done only when the action can be shown to be locally rigid in $\operatorname{Diff}^{\infty}(M)$ in the sense of Definition 1.1. This implicitly omits both the degree of regularity to which the perturbed and unperturbed actions are close and the degree of regularity with which the size of the conjugacy is small. In other words local rigidity is $C^{\infty, \infty, i, \infty, m}$ local rigidity for some unspecified $i$ and $m$, 
and I always fail to specify $i$ and $m$ even when they are known. Occasionally a result is stated that only produces a finite regularity conjugacy, with this issue only remarked on following the statement of the result. It seems quite likely that most results of this kind can be improved to produce $C^{\infty}$ conjugacies using the techniques of [FM2; FM3], see discussion at the end of Section 5.1.

Lastly we remark that the study of local rigidity of group actions is often closely intertwined with the study of global rigidity of group actions. The meaning of the phrase global rigidity is not entirely precise, but it is typically used to cover settings in which one can classify all group actions satisfying certain hypotheses on a certain manifold or class of manifolds. The study of global rigidity is too broad and interesting to summarize briefly, but some examples are mentioned below when they are closely related to work on local rigidity. See [FK; La] for recent surveys concerning both local and global rigidity.

\section{A brief digression: some examples of groups and actions}

In this section we briefly describe some of the groups that will play important roles in the results discussed here. The reader already familiar with semisimple Lie groups and their lattices may want to skip to the second subsection where we give descriptions of group actions.

2.1. Semisimple groups and their lattices. By a simple Lie group, we mean a connected Lie group all of whose normal subgroups are discrete, though we make the additional convention that $\mathbb{R}$ and $S^{1}$ are not simple. By a semisimple Lie group we mean the quotient of a product of simple Lie groups by some subgroup of the product of their centers. Note that with our conventions, the center of a simple Lie group is discrete and is in fact the maximal normal subgroup. There is an elaborate structure theory of semisimple Lie groups and the groups are completely classified, see $[\mathrm{He}]$ or $[\mathrm{Kn}]$ for details. Here we merely describe some examples, all of which are matrix groups. All connected semisimple Lie groups are discrete central extensions of matrix groups, so the reader will lose very little by always thinking of matrix groups.

(1) The groups $S L(n, \mathbb{R}), S L(n, \mathbb{C})$ and $S L(n, \mathbb{M})$ of $n$ by $n$ matrices of determinant one over the real numbers, the complex numbers or the quaternions.

(2) The group $S P(2 n, \mathbb{R})$ of $2 n$ by $2 n$ matrices of determinant one which preserve a real symplectic form on $\mathbb{R}^{2 n}$.

(3) The groups $S O(p, q), S U(p, q)$ and $S P(p, q)$ of matrices which preserve inner products of signature $(p, q)$ where the inner product is real linear on $\mathbb{R}^{p+q}$, hermitian on $\mathbb{C}^{p+q}$ or quaternionic hermitian on $\mathbb{M}^{p+q}$ respectively.

Let $G$ be a semisimple Lie group which is a subgroup of $G L(n, \mathbb{R})$. We say that $G$ has real rank $k$ if $G$ has a $k$ dimensional abelian subgroup which is conjugate 
to a subgroup of the real diagonal matrices and no $k+1$ dimensional abelian subgroups with the same property. The groups in (1) have rank $n-1$, the groups in (2) have rank $n$ and the groups in (3) have rank $\min (p, q)$.

Since this article focuses primarily on finitely generated groups, we are more interested in discrete subgroups of Lie groups than in the Lie groups themselves. A discrete subgroup $\Gamma$ in a Lie group $G$ is called a lattice if $G / \Gamma$ has finite Haar measure. The lattice is called cocompact or uniform if $G / \Gamma$ is compact and nonuniform or simply not cocompact otherwise. If $G=G_{1} \times \cdots \times G_{n}$ is a product then we say a lattice $\Gamma<G$ is irreducible if it's projection to each $G_{i}$ is dense. More generally we make the same definition for an almost direct product, by which we mean a direct product $G$ modulo some subgroup of the center $Z(G)$. Lattices in semisimple Lie groups can always be constructed by arithmetic methods, see [Bo] and also [Mr] for more discussion. In fact, one of the most important results in the theory of semisimple Lie groups is that if $G$ is a semisimple Lie group without compact factors, then all irreducible lattices in $G$ are arithmetic unless $G$ is locally isomorphic to $S O(1, n)$ or $S U(1, n)$. For $G$ of real rank at least two, this is Margulis' arithmeticity theorem, which he deduced from his superrigidity theorems [Ma2; Ma3; Ma4]. For nonuniform lattices, Margulis had an earlier proof which does not use the superrigidity theorems, see [Ma1; Ma2]. This earlier proof depends on the study of dynamics of unipotent elements on the space $G / \Gamma$, and particularly on what is now known as the "nondivergence of unipotent flows". Special cases of the superrigidity theorems were then proven for $\operatorname{Sp}(1, n)$ and $F_{4}^{-20}$ by Corlette and Gromov-Schoen, which sufficed to imply the statement on arithmeticity given above [Co2; GS]. As we will be almost exclusively concerned with arithmetic lattices, we do not give examples of nonarithmetic lattices here, but refer the reader to [Ma4] and $[\mathrm{Mr}]$ for more discussion. A formal definition of arithmeticity, at least when $G$ is algebraic is:

Definition 2.1. Let $G$ be a semisimple algebraic Lie group and $\Gamma<G$ a lattice. Then $\Gamma$ is arithmetic if there exists a semisimple algebraic Lie group $H$ defined over $\mathbb{Q}$ such that

(1) there is a homomorphism $\pi: H^{0} \rightarrow G$ with compact kernel,

(2) there is a rational structure on $H$ such that the projection of the integer points of $H$ to $G$ are commensurable to $\Gamma$, i.e. $\pi(H(\mathbb{Z})) \cap \Gamma$ is of finite index in both $H(\mathbb{Z})$ and $\Gamma$.

We now give some examples of arithmetic lattices. The simplest is to take the integer points in a simple (or semisimple) group $G$ which is a matrix group, e.g. $S L(n, \mathbb{Z})$ or $S p(n, \mathbb{Z})$. This exact construction always yields lattices, but also always yields nonuniform lattices. In fact the lattices one can construct in 
this way have very special properties because they will contain many unipotent matrices. If a lattice is cocompact, it will necessarily contain no unipotent matrices. The standard trick for understanding the structure of lattices in $G$ which become integral points after passing to a compact extension is called change of base. For much more discussion see [Ma4; Mr; Z2]. We give one example to illustrate the process. Let $G=S O(m, n)$ which we view as the set of matrices in $S L(n+m, \mathbb{R})$ which preserve the inner product

$$
\langle v, w\rangle=\left(-\sqrt{2} \sum_{i=1}^{m} v_{i} w_{i}\right)+\left(\sum_{i=m+1}^{n+m} v_{i} w_{i}\right)
$$

where $v_{i}$ and $w_{i}$ are the $i$ th components of $v$ and $w$. This form, and therefore $G$, are defined over the field $\mathbb{Q}(\sqrt{2})$ which has a Galois conjugation $\sigma$ defined by $\sigma(\sqrt{2})=-\sqrt{2}$. If we looks at the points $\Gamma=G(\mathbb{Z}[\sqrt{2}])$, we can define an embedding of $\Gamma$ in $S O(m, n) \times S O(m+n)$ by taking $\gamma$ to $(\gamma, \sigma(\gamma))$. It is straightforward to check that this embedding is discrete. In fact, this embeds $\Gamma$ in $H=S O(m, n) \times S O(m+n)$ as integral points for the rational structure on $H$ where the rational points are exactly the points $(m, \sigma(m))$ where $m \in G(\mathbb{Q}(\sqrt{2}))$. This makes $\Gamma$ a lattice in $H$ and it is easy to see that $\Gamma$ projects to a lattice in $G$, since $G$ is cocompact in $H$. What is somewhat harder to verify is that $\Gamma$ is cocompact in $H$, for which we refer the reader to the list of references above.

Similar constructions are possible with $S U(m, n)$ or $S P(m, n)$ in place of $S O(m, n)$ and also with more simple factors and fields with more Galois automorphisms. There are also a number of other constructions of arithmetic lattices using division algebras. See [Mr] for a comprehensive treatment.

We end this section by defining a key property of many semisimple groups and their lattices. This is property $(T)$ of Kazhdan, and was introduced by Kazhdan in [Ka1] in order to prove that nonuniform lattices in higher rank semisimple Lie groups are finitely generated and have finite abelianization. It has played a fundamental role in many subsequent developments. We do not give Kazhdan's original definition, but one which was shown to be equivalent by work of Delorme and Guichardet [De; Gu].

Definition 2.2. A group $\Gamma$ has property $(T)$ of Kazhdan if $H^{1}(\Gamma, \pi)=0$ for every continuous unitary representation $\pi$ of $\Gamma$ on a Hilbert space. This is equivalent to saying that any continuous isometric action of $\Gamma$ on a Hilbert space has a fixed point.

REMARKS. (1) Kazhdan's definition is that the trivial representation is isolated in the unitary dual of $\Gamma$.

(2) If a continuous group $G$ has property $(T)$ so does any lattice in $G$. This result was proved in [Ka1]. 
(3) Any semisimple Lie group has property $(T)$ if and only if it has no simple factors locally isomorphic to $S O(1, n)$ or $S U(1, n)$. For a discussion of this fact and attributions, see [HV]. For groups with all simple factors of real rank at least three, this is proven in [Ka1].

(4) No noncompact amenable group, and in particular no noncompact abelian group, has property $(T)$. An easy averaging argument shows that all compact groups have property $(T)$.

Groups with property $(T)$ play an important role in many areas of mathematics and computer science.

2.2. Some actions of groups and lattices. Here we define and give examples of the general classes of actions for which local rigidity results have been proven. Let $H$ be a Lie group and $L<H$ a closed subgroup. Then a diffeomorphism $f$ of $H / L$ is called affine if there is a diffeomorphism $\tilde{f}$ of $H$ such that $f([h])=$ $\tilde{f}(h)$ where $\tilde{f}=A \circ \tau_{h}$ with $A$ an automorphism of $H$ with $A(L)=L$ and $\tau_{h}$ is left translation by some $h$ in $H$. Two obvious classes of affine diffeomorphisms are left translations on any homogeneous space and either linear automorphisms of tori or more generally automorphisms of nilmanifolds. A group action is called affine if every element of the group acts by an affine diffeomorphism. It is easy to check that the full group of affine diffeomorphisms $\operatorname{Aff}(H / L)$ is a finite dimensional Lie group and an affine action of a group $D$ is a homomorphism $\pi: D \rightarrow \operatorname{Aff}(H / L)$. The structure of $\operatorname{Aff}(H / L)$ is surprisingly complicated in general, it is a quotient of a subgroup of the $\operatorname{group} \operatorname{Aut}(H) \ltimes H$ where $\operatorname{Aut}(H)$ is a the group of automorphisms of $H$. For a more detailed discussion of this relationship, see [FM1, Section 6]. While it is not always the case that any affine action of a group $D$ on $H / L$ can be described by a homomorphism $\pi$ : $D \rightarrow \operatorname{Aut}(H) \ltimes H$, this is true for two important special cases:

(1) $D$ is a connected semisimple Lie group and $L$ is a cocompact lattice in $H$,

(2) $D$ is a lattice in a semisimple Lie group $G$ where $G$ has no compact factors and no simple factors locally isomorphic to $S O(1, n)$ or $S U(1, n)$, and $L$ is a cocompact lattice in $H$.

These facts are [FM1, Theorem 6.4 and 6.5] where affine actions as in (1) and (2) above are classified.

The most obvious examples of affine actions of large groups are of the following forms, which are frequently referred to as standard actions:

(1) Actions of groups by automorphisms of nilmanifolds. I.e. let $N$ be a simply connected nilpotent group, $\Lambda<N$ a lattice (which is necessarily cocompact) and assume a finitely generated group $\Gamma$ acts by automorphisms of $N$ preserving $\Lambda$. The most obvious examples of this are when $N=\mathbb{R}^{n}, \Lambda=\mathbb{Z}^{n}$ and $\Gamma<S L(n, \mathbb{Z})$, in which case we have a linear action of $\Gamma$ on $\mathbb{T}^{n}$. 
(2) Actions by left translations. I.e. let $H$ be a Lie group and $\Lambda<H$ a cocompact lattice and $\Gamma<H$ some subgroup. Then $\Gamma$ acts on $H / \Lambda$ by left translations. Note that in this case $\Gamma$ need not be discrete.

(3) Actions by isometries. Here $K$ is a compact group which acts by isometries on some compact manifold $M$ and $\Gamma<K$ is a subgroup. Note that here $\Gamma$ is either discrete or a discrete extension of a compact group.

We now briefly define a few more general classes of actions, for which local rigidity results are either known or conjectured. We first fix some notations. Let $A$ and $D$ be topological groups, and $B<A$ a closed subgroup. Let $\rho$ : $D \times A / B \rightarrow A / B$ be a continuous affine action.

Definition 2.3. (1) Let $A, B, D$ and $\rho$ be as above. Let $C$ be a compact group of affine diffeomorphisms of $A / B$ that commute with the $D$ action. We call the action of $D$ on $C \backslash A / B$ a generalized affine action.

(2) Let $A, B, D$ and $\rho$ be as in 1 above. Let $M$ be a compact Riemannian manifold and $\iota: D \times A / B \rightarrow \operatorname{Isom}(M)$ a $C^{1}$ cocycle. We call the resulting skew product $D$ action on $A / B \times M$ a quasiaffine action. If $C$ and $D$ are as in 2, and $\alpha: D \times C \backslash A / B \rightarrow \operatorname{Isom}(M)$ is a $C^{1}$ cocycle, then we call the resulting skew product $D$ action on $C \backslash A / B \times M$ a generalized quasiaffine action.

For many of the actions we consider here, there will be a foliation of particular importance. If $\rho$ is an action of a group $D$ on a manifold $N$, and $\rho$ preserves a foliation $\mathfrak{F}$ and a Riemannian metric along the leaves of $\mathfrak{F}$, we call $\mathfrak{F}$ a central foliation for $\rho$. For quasiaffine and generalized quasiaffine actions on manifolds of the form $C \backslash A / B \times M$ the foliation by leaves of the $\{[a]\} \times M$ is always a central foliation. There are also actions with more complicated central foliations. For example if $H$ is a Lie group, $\Lambda<H$ is discrete and a subgroup $G<H$ acts on $H / \Lambda$ by left translations, then the foliation of $H / \Lambda$ by orbits of the centralizer $Z_{H}(G)$ of $G$ in $H$ is a central foliation. It is relatively easy to construct examples where this foliation has dense leaves. Another example of an action which has a foliation with dense leaves is to embed the $\mathbb{Z}[\sqrt{2}]$ points of $S O(m, n)$ into $S L(2(m+n), \mathbb{Z})$ as described in the preceding subsection and then let this group act on $\mathbb{T}^{2(m+n)}$ linearly. It is easy to see in this case that the maximal central foliation for the action is a foliation by densely embedded leaves none of which are compact.

We end this section by describing briefly the standard construction of an induced or suspended action. This notion can be seen as a generalization of the construction of a flow under a function or as an analogue of the more algebraic notion of inducing a representation. Given a group $H$, a (usually closed) subgroup $L$, and an action $\rho$ of $L$ on a space $X$, we can form the space $(H \times X) / L$ 
where $L$ acts on $H \times X$ by $h \cdot(l, x)=\left(l h^{-1}, \rho(h) x\right)$. This space now has a natural $H$ action by left multiplication on the first coordinate. Many properties of the $L$ action on $X$ can be studied more easily in terms of properties of the $H$ action on $(H \times X) / L$. This construction is particularly useful when $L$ is a lattice in $H$.

\section{Prehistory}

3.1. Local and global rigidity of homomorphisms into finite dimensional groups. The earliest work on local rigidity in the context of Definition 1.1 was contained in series of works by Calabi-Vesentini, Selberg, Calabi and Weil, which resulted in the following:

THEOREM 3.1. Let $G$ be a semisimple Lie group and assume that $G$ is not locally isomorphic to $S L(2, \mathbb{R})$. Let $\Gamma<G$ be an irreducible cocompact lattice, then the defining embedding of $\Gamma$ in $G$ is locally rigid.

REMARKS. (1) If $G=S L(2, \mathbb{R})$ the theorem is false and there is a large, well studied space of deformation of $\Gamma$ in $G$, known as the Teichmüller space.

(2) There is an analogue of this theorem for lattices that are not cocompact. This result was proven later and has a more complicated history which we omit here. In this case it is also necessary to exclude $G$ locally isomorphic to $S L(2, \mathbb{C})$.

This theorem was originally proven in special cases by Calabi, Calabi-Vesentini and Selberg. In particular, Selberg gives a proof for cocompact lattices in $S L(n, \mathbb{R})$ for $n \geq 3$ in [S], Calabi-Vesentini give a proof when the associated symmetric space $X=G / K$ is Kähler in [CV] and Calabi gives a proof for $G=S O(1, n)$ where $n \geq 3$ in [C]. Shortly afterwards, Weil gave a complete proof of Theorem 3.1 in [We1; We2].

In all of the original proofs, the first step was to show that any perturbation of $\Gamma$ was discrete and therefore a cocompact lattice. This is shown in special cases in $[\mathrm{C} ; \mathrm{CV} ; \mathrm{S}]$ and proven in a somewhat broader context than Theorem 3.1 in [W1].

The different proofs of cases of Theorem 3.1 are also interesting in that there are two fundamentally different sets of techniques employed and this dichotomy continues to play a role in the history of rigidity. Selberg's proof essentially combines algebraic facts with a study of the dynamics of iterates of matrices. He makes systematic use of the existence of singular directions, or Weyl chamber walls, in maximal diagonalizable subgroups of $S L(n, \mathbb{R})$. Exploiting these singular directions is essential to much later work on rigidity, both of lattices in higher rank groups and of actions of abelian groups. It seems possible to generalize Selberg's proof to the case of $G$ an $\mathbb{R}$-split semisimple Lie group with 
rank at least 2. Selberg's proof, which depended on asymptotics at infinity of iterates of matrices, inspired Mostow's explicit use of boundaries in his proof of strong rigidity [Mo2]. Mostow's work in turn provided inspiration for the use of boundaries in later work of Margulis, Zimmer and others on rigidity properties of higher rank groups.

The proofs of Calabi, Calabi-Vesentini and Weil involve studying variations of geometric structures on the associated locally symmetric space. The techniques are analytic and use a variational argument to show that all variations of the geometric structure are trivial. This work is a precursor to much work in geometric analysis studying variations of geometric structures and also informs later work on proving rigidity/vanishing of harmonic forms and maps. The dichotomy between approaches based on algebra/dynamics and approaches that are in the spirit of geometric analysis continues through much of the history of rigidity and the history of local rigidity of group actions in particular.

Shortly after completing this work, Weil discovered a new criterion for local rigidity [We3]. In the context of Theorem 3.1, this allows one to avoid the step of showing that a perturbation of $\Gamma$ remains discrete. In addition, this result opened the way for understanding local rigidity of more general representations of discrete groups.

THEOREM 3.2. Let $\Gamma$ be a finitely generated group, $G$ a Lie group and $\pi$ : $\Gamma \rightarrow G$ a homomorphism. Then $\pi$ is locally rigid if $H^{1}(\Gamma, \mathfrak{g})=0$. Here $\mathfrak{g}$ is the Lie algebra of $G$ and $\Gamma$ acts on $\mathfrak{g}$ by $A d_{G} \circ \pi$.

Weil's proof of this result uses only the implicit function theorem and elementary properties of the Lie group exponential map. The same theorem is true if $G$ is an algebraic group over any local field of characteristic zero. In [We3], Weil remarks that if $\Gamma<G$ is a cocompact lattice and $G$ satisfies the hypothesis of Theorem 3.1, then the vanishing of $H^{1}(\Gamma, \mathfrak{g})$ can be deduced from the computations in [We2]. The vanishing of $H^{1}(\Gamma, \mathfrak{g})$ is proven explicitly by Matsushima and Murakami in [MM].

Motivated by Weil's work and other work of Matsushima, conditions for vanishing of $H^{1}(\Gamma, \mathfrak{g})$ were then studied by many authors. See particularly $[\mathrm{MM}]$ and $[\mathrm{Rg} 1]$. The results in these papers imply local rigidity of many linear representations of lattices.

To close this section, I will briefly discuss some subsequent developments concerning rigidity of lattices in Lie groups that motivated the study of both local and global rigidity of group actions.

The first remarkable result in this direction is Mostow's rigidity theorem, see [Mo1] and references there. Given $G$ as in Theorem 3.1, and two irreducible cocompact lattices $\Gamma_{1}$ and $\Gamma_{2}$ in $G$, Mostow proves that any isomorphism from $\Gamma_{1}$ to $\Gamma_{2}$ extends to an isomorphism of $G$ with itself. Combined with the prin- 
cipal theorem of [We1] which shows that a perturbation of a lattice is again a lattice, this gives a remarkable and different proof of Theorem 3.1, and Mostow was motivated by the desire for a "more geometric understanding" of Theorem 3.1 [Mo1]. Mostow's theorem is in fact a good deal stronger, and controls not only homomorphisms $\Gamma \rightarrow G$ near the defining homomorphism, but any homomorphism into any other simple Lie group $G^{\prime}$ where the image is lattice. As mentioned above, Mostow's approach was partially inspired by Selberg's proof of certain cases of Theorem 3.1, [Mo2]. A key step in Mostow's proof is the construction of a continuous map between the geometric boundaries of the symmetric spaces associated to $G$ and $G^{\prime}$. Boundary maps continue to play a key role in many developments in rigidity theory. A new proof of Mostow rigidity, at least for $G_{i}$ of real rank one, was provided by Besson, Courtois and Gallot. Their approach is quite different and has had many other applications concerning rigidity in geometry and dynamics; see [BCG; $\mathrm{CF}]$, for example.

The next remarkable result in this direction is Margulis' superrigidity theorem. Margulis proved this theorem as a tool to prove arithmeticity of irreducible uniform lattices in groups of real rank at least 2. For irreducible lattices in semisimple Lie groups of real rank at least 2, the superrigidity theorems classifies all finite dimensional linear representations. Margulis' theorem holds for irreducible lattices in semisimple Lie groups of real rank at least two. Given a lattice $\Gamma<G$ where $G$ is simply connected, one precise statement of some of Margulis results is to say that any linear representation $\sigma$ of $\Gamma$ almost extends to a linear representation of $G$. By this we mean that there is a linear representation $\tilde{\sigma}$ of $G$ and a bounded image representation $\bar{\sigma}$ of $\Gamma$ such that $\sigma(\gamma)=\tilde{\sigma}(\gamma) \bar{\sigma}(\gamma)$ for all $\gamma$ in $G$. Margulis' theorems also give an essentially complete description of the representations $\bar{\sigma}$, up to some issues concerning finite image representations. The proof here is partially inspired by Mostow's work: a key step is the construction of a measurable "boundary map". However the methods for producing the boundary map in this case are very dynamical. Margulis' original proof used Oseledec Multiplicative Ergodic Theorem. Later proofs were given by both Furstenberg and Margulis using the theory of group boundaries as developed by Furstenberg from his study of random walks on groups [Fu1; Fu2]. Furstenberg's probabilistic version of boundary theory has had a profound influence on many subsequent developments in rigidity theory. For more discussion of Margulis' superrigidity theorem, see [Ma2; Ma3; Ma4].

A main impetus for studying rigidity of group actions on manifolds came from Zimmer's theorem on superrigidity for cocycles. This theorem and it's proof were strongly motivated by Margulis' work. In fact, Margulis' theorem is Zimmer's theorem for a certain cocycle $\alpha: G \times G / \Gamma \rightarrow \Gamma$ and the proof of Zimmer's theorem is quite similar to the proof of Margulis'. In order to avoid 
technicalities, we describe only a special case of this result. Let $M$ be a compact manifold, $H$ a matrix group and $P=M \times H$. Now let a group $\Gamma$ act on $M$ and $P$ continuously, so that the projection from $P$ to $M$ is equivariant. Further assume that the action on $M$ is measure preserving and ergodic. If $\Gamma$ is a lattice in a simply connected, semisimple Lie group $G$ all of whose simple factors have real rank at least two then there is a measurable map $s: M \rightarrow H$, a representation $\pi: G \rightarrow H$, a compact subgroup $K<H$ which commutes with $\pi(G)$ and a measurable map $\Gamma \times M \rightarrow K$ such that

$$
\gamma \cdot s(m)=k(m, \gamma) \pi(\gamma) s(\gamma \cdot m) .
$$

It is easy to check from this equation that the map $K$ satisfies a certain equation that makes it into a cocycle over the action of $\Gamma$. One should view $s$ as providing coordinates on $P$ in which the $\Gamma$ action is almost a product. For more discussion of this theorem the reader should see any of [Fe1; Fe2; FM1; Fu3; Z2]. (The version stated here is only proven in [FM1], previous proofs all yielded somewhat more complicated statements.) As a sample application, let $M=\mathbb{T}^{n}$ and let $P$ be the frame bundle of $M$, i.e. the space of frames in the tangent bundle of $M$. Since $\mathbb{T}^{n}$ is parallelizable, we have $P=\mathbb{T}^{n} \times G L\left(n, \mathbb{R}^{n}\right)$. The cocycle superrigidity theorem then says that "up to compact noise", the derivative of any measure preserving $\Gamma$ action on $\mathbb{T}^{n}$ looks measurably like a constant linear map. In fact, the cocycle superrigidity theorems apply more generally to continuous actions on any principal bundle $P$ over $M$ with fiber $H$, an algebraic group, and in this context produces a measurable section $s: M \rightarrow P$ satisfying equation (3-1). So in fact, cocycle superrigidity implies that for any action preserving a finite measure on any manifold the derivative cocycle looks measurably like a constant cocycle, up to compact noise. That cocycle superrigidity provides information about actions of groups on manifolds through the derivative cocycle was first observed in [Fu3]. Zimmer originally proved cocycle superrigidity in order to study orbit equivalence of group actions. For a recent survey of subsequent developments concerning orbit equivalence rigidity and other forms of superrigidity for cocycles, see [S12].

3.2. Stability in hyperbolic dynamics. A diffeomorphism $f$ of a manifold $X$ is said to be Anosov if there exists a continuous $f$ invariant splitting of the tangent bundle $T X=E_{f}^{u} \oplus E_{f}^{s}$ and constants $a>1$ and $C, C^{\prime}>0$ such that for every $x \in X$,

(1) $\left\|D f^{n}\left(v^{u}\right)\right\| \geq C a^{n}\left\|v^{u}\right\|$ for all $v^{u} \in E_{f}^{u}(x)$ and,

(2) $\left\|D f^{n}\left(v^{s}\right)\right\| \leq C^{\prime} a^{-n}\left\|v^{s}\right\|$ for all $v^{s} \in E_{f}^{s}(x)$.

We note that the constants $C$ and $C^{\prime}$ depend on the choice of metric, and that a metric can always be chosen so that $C=C^{\prime}=1$. There is an analogous notion 
for a flow $f_{t}$, where $T X=T \mathscr{O} \oplus E_{f_{t}}^{u} \oplus E_{f_{t}}^{s}$ where $T \mathcal{O}$ is the tangent space to the flow direction and vectors in $E_{f_{t}}^{u}$ (resp. $E_{f_{t}}^{s}$ ) are uniformly expanded (resp. uniformly contracted) by the flow. This notion was introduced by Anosov and named after Anosov by Smale, who popularized the notion in the United States [An; Sm]. One of the earliest results in the subject is Anosov's proof that Anosov diffeomorphisms are structurally stable, or, in our language $C^{1,1,1,0,0}$ locally rigid. There is an analogous result for flows, though this requires that one introduce a notion of time change that we will not consider here. Since Anosov also showed that $C^{2}$ Anosov flows and diffeomorphisms are ergodic, structural stability implies that the existence of an open set of "chaotic" dynamical systems.

The notion of an Anosov diffeomorphism has had many interesting generalizations, for example: Axiom A diffeomorphisms, nonuniformly hyperbolic diffeomorphisms, and diffeomorphisms admitting a dominated splitting. The notion that has been most useful in the study of local rigidity is the notion of a partially hyperbolic diffeomorphism as introduced by Hirsch, Pugh and Shub. Under strong enough hypotheses, these diffeomorphisms have a weaker stability property similar to structural stability. More or less, the diffeomorphisms are hyperbolic relative to some foliation, and any nearby action is hyperbolic to some nearby foliation. To describe more precisely the class of diffeomorphisms we consider and the stability property they enjoy, we require some definitions.

The use of the word foliation varies with context. Here a foliation by $C^{k}$ leaves will be a continuous foliation whose leaves are $C^{k}$ injectively immersed submanifolds that vary continuously in the $C^{k}$ topology in the transverse direction. To specify transverse regularity we will say that a foliation is transversely $C^{r}$. A foliation by $C^{k}$ leaves which is tranversely $C^{k}$ is called simply a $C^{k}$ foliation. (Note our language does not agree with that in the reference [HPS].)

Given an automorphism $f$ of a vector bundle $E \rightarrow X$ and constants $a>b \geq 1$, we say $f$ is $(a, b)$-partially hyperbolic or simply partially hyperbolic if there is a metric on $E$, a constant and $C \geq 1$ and a continuous $f$ invariant, nontrivial splitting $E=E_{f}^{u} \oplus E_{f}^{c} \oplus E_{f}^{s}$ such that for every $x$ in $X$ :

(1) $\left\|f^{n}\left(v^{u}\right)\right\| \geq C a^{n}\left\|v^{u}\right\|$ for all $v^{u} \in E_{f}^{u}(x)$,

(2) $\left\|f^{n}\left(v^{s}\right)\right\| \leq C^{-1} a^{-n}\left\|v^{s}\right\|$ for all $v^{s} \in E_{f}^{s}(x)$ and

(3) $C^{-1} b^{-n}\left\|v^{0}\right\|<\left\|f^{n}\left(v^{0}\right)\right\| \leq C b^{n}\left\|v^{0}\right\|$ for all $v^{0} \in E_{f}^{c}(x)$ and all integers $n$.

A $C^{1}$ diffeomorphism $f$ of a manifold $X$ is $(a, b)$-partially hyperbolic if the derivative action $D f$ is $(a, b)$-partially hyperbolic on $T X$. We remark that for any partially hyperbolic diffeomorphism, there always exists an adapted metric for which $C=1$. Note that $E_{f}^{c}$ is called the central distribution of $f, E_{f}^{u}$ is called the unstable distribution of $f$ and $E_{f}^{s}$ the stable distribution of $f$. 
Integrability of various distributions for partially hyperbolic dynamical systems is the subject of much research. The stable and unstable distributions are always tangent to invariant foliations which we call the stable and unstable foliations and denote by $\mathcal{W}_{f}^{s}$ and $\mathcal{W}_{f}^{u}$. If the central distribution is tangent to an $f$ invariant foliation, we call that foliation a central foliation and denote it by $\mathcal{W}_{f}^{c}$. If there is a unique foliation tangent to the central distribution we call the central distribution uniquely integrable. For smooth distributions unique integrability is a consequence of integrability, but the central distribution is usually not smooth. If the central distribution of an $(a, b)$-partially hyperbolic diffeomorphism $f$ is tangent to an invariant foliation $\mathcal{W}_{f}^{c}$, then we say $f$ is $r$-normally hyperbolic to $W_{f}^{c}$ for any $r$ such that $a>b^{r}$. This is a special case of the definition of $r$-normally hyperbolic given in [HPS].

Before stating a version of one of the main results of [HPS], we need one more definition. Given a group $G$, a manifold $X$, two foliations $\mathfrak{F}$ and $\mathfrak{F}^{\prime}$ of $X$, and two actions $\rho$ and $\rho^{\prime}$ of $G$ on $X$, such that $\rho$ preserves $\mathfrak{F}$ and $\rho^{\prime}$ preserves $\mathfrak{F}^{\prime}$, following [HPS] we call $\rho$ and $\rho^{\prime}$ leaf conjugate if there is a homeomorphism $h$ of $X$ such that:

(1) $h(\mathfrak{F})=\mathfrak{F}^{\prime}$ and

(2) for every leaf $\mathfrak{L}$ of $\mathfrak{F}$ and every $g \in G$, we have $h(\rho(g) \mathfrak{L})=\rho^{\prime}(g) h(\mathfrak{L})$.

The map $h$ is then referred to as a leaf conjugacy between $(X, \mathfrak{F}, \rho)$ and $\left(X, \mathfrak{F}^{\prime}, \rho^{\prime}\right)$. This essentially means that the actions are conjugate modulo the central foliations.

We state a special case of some the results of Hirsch-Pugh-Shub on perturbations of partially hyperbolic actions of $\mathbb{Z}$, see [HPS]. There are also analogous definitions and results for flows. As these are less important in the study of local rigidity, we do not discuss them here.

THEOREM 3.3. Let $f$ be an $(a, b)$-partially hyperbolic $C^{k}$ diffeomorphism of a compact manifold $M$ which is $k$-normally hyperbolic to a $C^{k}$ central foliation $\mathcal{W}_{f}^{c}$. Then for any $\delta>0$, if $f^{\prime}$ is a $C^{k}$ diffeomorphism of $M$ which is sufficiently $C^{1}$ close to $f$ we have the following:

(1) $f^{\prime}$ is $\left(a^{\prime}, b^{\prime}\right)$-partially hyperbolic, where $\left|a-a^{\prime}\right|<\delta$ and $\left|b-b^{\prime}\right|<\delta$, and the splitting $T M=E_{f^{\prime}}^{u} \oplus E_{f^{\prime}}^{c} \oplus E_{f^{\prime}}^{s}$ for $f^{\prime}$ is $C^{0}$ close to the splitting for $f$;

(2) there exist $f^{\prime}$ invariant foliations by $C^{k}$ leaves $\mathscr{W}_{f^{\prime}}^{c}$ tangent to $E_{f^{\prime}}^{c}$, which is close in the natural topology on foliations by $C^{k}$ leaves to $\mathscr{W}_{f}^{c}$,

(3) there exists a (nonunique) homeomorphism $h$ of $M$ with $h\left(W_{f}^{c}\right)=W_{f^{\prime}}^{c}$, and $h$ is $C^{k}$ along leaves of $W_{f}^{c}$, furthermore $h$ can be chosen to be $C^{0}$ small and $C^{k}$ small along leaves of $\mathcal{W}_{f}^{c}$ 
(4) the homeomorphism $h$ is a leaf conjugacy between the actions $\left(M, \mathscr{W}_{f}^{c}, f\right)$ and $\left(M, W_{f^{\prime}}^{c}, f^{\prime}\right)$.

Conclusion (1) is easy and probably older than [HPS]. One motivation for Theorem 3.3 is to study stability of dynamical properties of partially hyperbolic diffeomorphisms. See the survey, [BPSW], for more discussion of that and related issues.

\section{History}

In this section, we describe the history of the subject roughly to the year 2000. More recent developments will be discussed below. Here we do not treat the subject entirely chronologically, but break the discussion into four subjects: first, the study of local rigidity of volume preserving actions, second the study of local rigidity of certain non-volume-preserving actions called boundary actions, third the existence of (many) deformations of (many) actions of groups that are typically quite rigid, and lastly a brief discussion of infinitesimal rigidity. This is somewhat ahistorical as the first results on smooth conjugacy of perturbations of group actions appear in [Gh1], which we describe in subsection 4.2. While those results are not precisely local rigidity results, they are clearly related and the techniques involved inform some later approaches to local rigidity.

4.1. Volume preserving actions. In this subsection, we discuss local rigidity of volume preserving actions. The acting groups will usually be lattices in higher rank semisimple Lie groups or higher rank free abelian groups. Many of the results discussed here were motivated by conjectures of Zimmer in [Z4; Z5]. The first result we mention, due to Zimmer, does not prove local rigidity, but did motivate much later work on the subject.

THEOREM 4.1. Let $\Gamma$ be a group with property $(T)$ of Kazhdan and let $\rho$ be a Riemannian isometric action of $\Gamma$ on a compact manifold $M$. Further assume the action is ergodic. Then any $C^{k}$ action $\rho^{\prime}$ which is $C^{k}$ close to $\rho$, volume preserving and ergodic, preserves a $C^{k-3}$ Riemannian metric.

REMARKS. (1) Zimmer first proved this theorem in [Z1], but only for $\Gamma$ a lattice in a semisimple group, all of whose simple factors have real rank at least two, and only producing a $C^{0}$ invariant metric for $\rho^{\prime}$. In [Z2], he gave the proof of the regularity stated here and in [Z4] he extended the theorem to all Kazhdan groups.

(2) In this theorem if $\rho^{\prime}$ is $C^{\infty}$, the invariant metric for $\rho^{\prime}$ can also be chosen $C^{\infty}$.

The first major result that actually produced a conjugacy between the perturbed and unperturbed actions was due to Hurder, [H1]. Again, this result is not quite a 
local rigidity theorem, but only a deformation rigidity theorem. Hurder's work is the first place where hyperbolic dynamics is used in the theory, and is the beginning of a long development in which hyperbolic dynamics play a key role.

THEOREM 4.2. The standard action of any finite index subgroup of $S L(n, \mathbb{Z})$ on the $n$ dimensional torus is deformation rigid when $n \geq 3$.

Hurder actually proves a much more general result. His theorem proves deformation rigidity of any group $\Gamma$ acting on the $n$ dimensional torus by linear transformations provided:

(1) the set of periodic points for the $\Gamma$ action is dense, and

(2) the first cohomology of any finite index subgroup of $\Gamma$ in any $n$ dimensional representation vanishes, and

(3) the action contains "enough" Anosov elements.

Here we intentionally leave the meaning of (3) vague, as the precise notion needed by Hurder is quite involved. To produce a continuous path of continuous conjugacies, Hurder only need conditions (1) and (2) and the existence of an Anosov diffeomorphism in the stabilizer of every periodic point for the action. The additional Anosov elements needed in (3) are used to improve regularity of the conjugacy, and better techniques for this are now available. Hurder's work has some applications to actions of irreducible lattices in products of rank 1 Lie groups, which we discuss below in subsection 6.2. These applications do not appear to be accessible by later techniques.

A key element in Hurder's argument is to use results of Stowe on persistence of fixed points under perturbations of actions [St1; St2]. To use Stowe's result one requires that the cohomology in the derivative representation at the fixed point vanishes. This is where (2) above is used. Hurder constructs his conjugacy by using the theorem of Anosov, mentioned above in subsection 3.2, that any Anosov diffeomorphism is structurally stable. This produces a conjugacy $h$ for an Anosov element $\rho\left(\gamma_{0}\right)$ which one then needs to see is a conjugacy for the entire group action. Hurder uses Stowe's results to show that $h$ is conjugacy for the $\Gamma$ actions at all of the periodic points for the $\Gamma$ action, and since periodic points are dense it is then a conjugacy for the full actions. The precise argument using Stowe's theorem is quite delicate and we do not attempt to summarize it here. This argument applies much more generally, see [H1, Theorem 2.9]. That the conjugacy depends continuously, and in fact even smoothly, on the original action is deduced from results on hyperbolic dynamics in [dlLMM].

The first major development after Hurder's theorem was a theorem of Katok and Lewis [KL1] of which we state a special case:

THEOREM 4.3. Let $\Gamma<S L(n, \mathbb{Z})$ be a finite index subgroup, $n>3$. Then the linear action of $\Gamma$ on $\mathbb{T}^{n}$ is locally rigid. 
It is worth noting that this theorem does not cover the case of $n=3$. A major ingredient in the proof is studying conjugacies produced by hyperbolic dynamics for certain $\mathbb{Z}$ actions generated by hyperbolic and partially hyperbolic diffeomorphisms in both the original action and the perturbation. The strategy is to find a hyperbolic generating set and to show that the conjugacies produced by the stability of those diffeomorphisms agree. A key ingredient idea is to show that they agree on the set of periodic orbits. Periodic orbits are then studied via Theorem 3.3 for elements of $\Gamma$ with large centralizers and large central foliations. Periodic orbits are detected as intersections of central foliations for different elements, and this allows the authors to show that the structure of the periodic set persists under deformations. Elements with large centralizers had previously been exploited by Lewis for studying infinitesimal rigidity of similar actions. The authors also exploit their methods to prove the following remarkable result:

THEOREM 4.4. Let $\mathbb{Z}^{n}$ be a maximal diagonalizable (over $\mathbb{R}$ ) subgroup of $S L(n+1, \mathbb{Z})$ where $n \geq 2$. The linear action of $\mathbb{Z}^{n}$ on $\mathbb{T}^{n+1}$ is locally rigid.

This result is the first in a long series of results showing that many actions of higher rank abelian groups are locally rigid. See Theorems 4.6 and 5.8 below for more instances of this remarkable behavior.

The next major development occurs in a paper of Katok, Lewis and Zimmer where Theorem 4.3 is extended to cover the case of $n=3$ as well as some more general groups acting on tori. Though this does not seem, on the face of it, to be a very dramatic development, an important idea is introduced in this paper. The authors proceed by comparing the measurable data coming from cocycle superrigidity to the continuous data provided by hyperbolic dynamics. In this context, this essentially allows the authors to show that the map $s$ in equation (3-1), described in the statement of cocycle superrigidity given in subsection 3.1 , is continuous. This idea of comparing the output of cocycle superrigidity to information provided by hyperbolic dynamics has played a major role in the development of both local and global rigidity of group actions.

The results in the papers [H1; KL1; KLZ] are all proven for particular actions of particular groups, and in particular are all proven for actions on tori. The next sequence of developments was a generalization of the ideas and methods contained in these papers to fairly general Anosov actions of higher rank lattices on nilmanifolds. Part of this development takes place in the works [Q1; Q2; QY]. A key difficulty in generalizing the early approaches to rigidity of groups of toral automorphims is in adapting the methods from hyperbolic dynamics which are used to improve the regularity of the conjugacy. In [KS1; KS2], Katok and Spatzier developed a broadly applicable method for smoothing conjugacies which depends on the theory of nonstationary normal forms as developed by Guysinsky and Katok in [GK; G]. Two main consequences of this method are: 
THEOREM 4.5. Let $G$ be a semisimple Lie group with all simple factors of real rank at least two, $\Gamma<G$ a lattice, $N$ a nilpotent Lie group and $\Lambda<N$ a lattice. Then any affine Anosov action of $\Gamma$ on $N / \Lambda$ is locally rigid.

Here by Anosov action, we mean that some element of $\Gamma$ acts on $N / \Lambda$ as an Anosov diffeomorphism.

THEOREM 4.6. Let $N$ a nilpotent Lie group and $\Lambda<N$ a lattice. Let $\mathbb{Z}^{d}$ be a group of affine transformations of $N / \Lambda$ such that the derivative action (on a subgroup of finite index) is simultaneously diagonalizable over $\mathbb{R}$ with no eigenvalues on the unit circle (i.e. on subgroup of finite index, each element of $\mathbb{Z}^{d}$ is an Anosov diffeomorphism which has semisimple derivative). Then the $\mathbb{Z}^{d}$ action on $N / \Lambda$ is locally rigid.

Katok and Spatzier also apply their method to show that for certain standard Anosov $\mathbb{R}^{d}$ actions the orbit foliation is locally rigid. I.e. any nearby action has conjugate orbit foliation. This yields interesting applications to rigidity of boundary actions, see Theorem 4.16 below. Also, combined with other results of the same authors on rigidity of cocycles over actions of Abelian groups, this yields local rigidity of certain algebraic actions of $\mathbb{R}^{d}$, [KS3; KS4]. We state a special case of these results here:

THEOREM 4.7. Let $G$ be an $\mathbb{R}$-split semisimple Lie group of real rank at least two. Let $\Lambda<G$ be a cocompact lattice and let $\mathbb{R}^{d}<G$ be a maximal $\mathbb{R}$-split subgroup. Then the $\mathbb{R}^{d}$ action on $G / \Lambda$ is locally rigid.

REMARKS. (1) Here "local rigidity" has a slightly different meaning than above. Since the automorphism group of $\mathbb{R}^{d}$ has nontrivial connected component, it is possible to perturb the action by taking a small automorphism of $\mathbb{R}^{d}$. What is proven in this theorem is that any small enough perturbation is conjugate to one obtained in this way.

(2) Another approach to related cocycle rigidity results is developed in the paper [KNT].

(3) The actual theorem in [KS2] is much more general.

A key ingredient in the Katok-Spatzier method is to find foliations which are orbits of transitive, isometric, smooth group actions for both the perturbed and unperturbed action. To show smoothness of the conjugacy, one constructs such group actions that

(1) are intertwined by a continuous conjugacy and

(2) exist on enough foliations to span all directions in the space.

This proves that the conjugacy is "smooth along many directions" and one then uses a variety of analytic methods to prove that the conjugacy is actually globally 
smooth. The fact that the transitive group exists and acts smoothly on the leaves of some foliation for the unperturbed action is typically obvious. One then uses the continuous conjugacy to define the group action along leaves for the perturbed action and the fact that the resulting action is smooth along leaves is verified using the normal form theory. The foliations along which one builds transitive group actions are typically central foliations for certain special elements of the suspension of the action. If the original action is a $\mathbb{Z}^{k}$ action by automorphisms on some nilmanifold $N / \Lambda$, the suspension of the action is the left action of $\mathbb{R}^{k}$ on the solv-manifold $M=\left(\mathbb{R}^{k} \ltimes N\right) /\left(\mathbb{Z}^{k} \ltimes \Lambda\right)$. A typical one parameter subgroup of $\mathbb{R}^{k}$ acts hyperbolically $M$, but certain special directions in $\mathbb{R}^{k}$, those in so-called Weyl chamber walls give rise to one parameter subgroups with nontrivial central direction. A key fact used in the argument is that one can find another subgroup of $\mathbb{R}^{k}$ for which the central foliation for some one parameter subgroup $\rho(t)$ is also a dynamically defined, contracting foliation for the some other element $a \in \mathbb{R}^{k}$.

All results quoted so far have strong assumptions on hyperbolicity of the action. For actions of semisimple groups and their lattices, the ultimate result on local rigidity in hyperbolic context was proven by Margulis and Qian in [MQ]. This result is for so-called weakly hyperbolic actions, which we define below. This work proceeds by first using a comparison between hyperbolic data and data from cocycle superrigidity to produce a continuous conjugacy between $C^{1}$ close actions and then uses an adaptation of the Katok-Spatzier smoothing method mentioned above. A key technical innovation in this work is the choice of cocycle to which cocycle superrigidity is applied. In all work to this point, it was applied to the derivative cocycle. Here it is applied to a cocycle that measures the difference between the action and the perturbation. To illustrate the idea, we give the definition of this cocycle, which we refer to as the Margulis-Qian cocycle, in the special case of actions by left translations. As this construction is quite general, we will let $D$ be the acting group. Let the $D$ action $\rho$ on $H / \Lambda$ be defined via a homomorphism $\pi_{0}: D \rightarrow H$. Let $\rho^{\prime}$ be a perturbation of $\rho$. If $D$ is connected it is clear that the action lifts to $\tilde{H}$ and therefore to $H$. If $D$ is discrete, this lifting still occurs, since the obstacle to lifting is a cohomology class in $H^{2}\left(D, \pi_{1}(H / \Lambda)\right)$ which does not change under a small perturbation of the action. (A direct justification without reference to group cohomology can be found in [MQ] section 2.3.) Write the lifted actions of $D$ on $H$ by $\tilde{\rho}$ and $\tilde{\rho}^{\prime}$ respectively. We can now define a cocycle $\alpha: D \times H \rightarrow H$ by

$$
\tilde{\rho}^{\prime}(g) x=\alpha(g, x) x
$$

for any $g$ in $D$ and any $x$ in $H$. It is easy to check that this is a cocycle and that it is right $\Lambda$ invariant, and so defines a cocycle $\alpha: D \times H / \Lambda \rightarrow H$. See [MQ] section 
2 or [FM1] section 6 for more discussion as well as for more general variants on this definition. We remark that the use of this cocycle allowed Margulis and Qian to prove the first local rigidity results for volume preserving actions of lattices that have no global fixed point. The construction of this cocycle is inspired by a cocycle used by Margulis in his first proof of superrigidity. This is the cocycle $G \times G / \Gamma \rightarrow \Gamma$ defined by the choice of a fundamental domain for $\Gamma$ in $G$. See Example 4 in subsection 6.2 for a more explicit description.

The work of Margulis and Qian applies to actions which satisfy the following condition. This condition essentially says that the action is hyperbolic in all possible directions, at least for some element of the acting group. It is easy to construct weakly hyperbolic actions of lattices, in particular $\Gamma$ acting on $G / \Lambda$ where $G$ is a simple Lie group and $\Lambda<G$ is a cocompact lattice and $\Gamma$ is any other lattice in $G$. It is important to note that for this example, no element acts as an Anosov diffeomorphism and, with an appropriate choice of $\Gamma$ and $\Lambda$, there are no finite $\Gamma$ orbits.

DEFINITION 4.8. An action $\rho$ of a group $D$ on a manifold $M$ is called weakly hyperbolic if there exist elements $d_{1}, \ldots, d_{k}$ and constants $a_{i}>b_{i} \geq 1$ for $i=$ $1, \ldots, k$ such that each $\rho\left(d_{i}\right)$ is $\left(a_{i}, b_{i}\right)$-partially hyperbolic in the sense of subsection 3.2 and we have $T M=\sum E_{\rho\left(d_{i}\right)}^{s}$. I.e. there are partially hyperbolic elements whose stable (or unstable) directions span the tangent space at any point.

THEOREM 4.9. Let $H$ be a real algebraic Lie group and $\Lambda<H$ a cocompact lattice. Assume $G$ is a semisimple Lie group with all simple factors of real rank at least two and $\Gamma<G$ is a lattice. Then any weakly hyperbolic, affine algebraic action of $\Gamma$ or $G$ on $H / \Lambda$ is locally rigid.

REMARK. This is somewhat more general than the result claimed in [MQ], as they only work with certain special classes of affine actions, which they call standard. This result can proven by the methods of [MQ], and a proof in precisely this generality can be read out of [FM1; FM3], simply by assuming that the common central direction for the acting group is trivial.

The next major result was a remarkable theorem of Benveniste concerning isometric actions. This is a stronger result than Theorem 4.1 because it actually produces a conjugacy, but is weaker in that it requires much stronger assumptions on the acting group.

THEOREM 4.10. Let $\Gamma$ be a cocompact lattice in a semisimple Lie group with all simple factors of real rank at least two. Let $\rho$ be an isometric $\Gamma$ action on a compact manifold $M$. Then $\rho$ is locally rigid. 
The proof of this theorem is inspired by the work of Calabi, Vesentini and Weil in the original proofs of Theorem 3.1 and is based on showing that certain deformations of foliated geometric structures are trivial. The argument is much more difficult than the classical case and uses Hamilton's implicit function theorem. This is the first occasion on which analytic methods like KAM theory or hard implicit function theorems appear in work on local rigidity of group actions. More recently these kinds of methods have been applied more systematically, see subsection 5.2.

The theorems described so far concern actions that are either isometric or weakly hyperbolic. There are many affine actions which satisfy neither of these dynamical hypotheses, but are genuinely partially hyperbolic. Local rigidity results for actions of this kind first arise in work of Nitica and Torok. We state special cases of two of their theorems:

THEOREM 4.11. Let $\Gamma<S L(n, \mathbb{Z})$ be a finite index subgroup with $n \geq 3$. Let $\rho_{1}$ be the standard $\Gamma$ action on $\mathbb{T}^{n}$ and let $\rho$ be the diagonal $\Gamma$ action on $\mathbb{T}^{n} \times \mathbb{T}^{m}$ defined by $\rho_{1}$ on the first factor and the trivial action on the second factor. The action $\rho$ is deformation rigid.

THEOREM 4.12. Let $\Gamma, \rho_{1}, \rho$ be as above and further assume that $m=1$. The action $\rho$ is locally rigid.

REMARKS. (1) Nitica and Torok prove more general theorems in which both $\Gamma$ and $\rho_{1}$ can be more general. The exact hypotheses required are different in the two theorems.

(2) We are being somewhat ahistorical here, Theorem 4.11 predates the work of Margulis and Qian.

(3) The conjugacy produced in the papers [NT1; NT2; T] is never $C^{\infty}$, but only $C^{k}$ for some choice of $k$. The choice of $k$ is essentially free and determines the size of perturbations or deformations that can be considered. It should be possible to produce a $C^{\infty}$ conjugacy by combining the arguments in these papers with arguments in [FM2; FM3], see the end of subsection 5.1 for some discussion.

The work of Nitica and Torok is quite complex, using several different ideas. The most novel is to study rigidity of cocycles over hyperbolic dynamical systems taking values in diffeomorphism groups. The dynamical system is either the action $\rho_{1}$ in Theorem 4.11 or 4.12 or it's restriction to any sufficiently generic subgroup containing an Anosov diffeomorphism of $\mathbb{T}^{n}$, and the target group is the group of diffeomorphisms of $\mathbb{T}^{m}$. This part of the work is inspired by a classical theorem of Livsic and the proof his modelled on his proof. To reduce the rigidity question to the cocycle question is quite difficult and depends on an adaptation of the work of [HPS] discussed in subsection 3.2 as well as use of 
results of Stowe [St1; St2]. Regrettably, the technology seems to limit the applicability of the ideas to diagonal actions $\rho_{1} \times \operatorname{Id}$ on products $M \times N$ where the action on $M$ has many periodic points and the action on $N$ is trivial. Theorem 4.12 also depends on the acting group having property $(T)$ of Kazhdan. The method of proof of Theorem 4.11 has additional applications, see particularly subsection 6.2 below.

To close this section, we remark that local rigidity is often considerably easier in the analytic setting. Not much work has been done in this direction, but there is an interesting note of Zeghib $[\mathrm{Zg}]$. A sample result is the following:

THEOREM 4.13. Let $\Gamma<S L(n, \mathbb{Z})$ be a subgroup of finite index and let $\rho$ be the standard action of $\Gamma$ on $\mathbb{T}^{n}$. Then any analytic action close enough to $\rho$ is analytically conjugate to $\rho$. Furthermore, if $M$ is a compact analytic manifold on which $\Gamma$ acts trivially and we let $\tilde{\rho}$ be the diagonal action of $\Gamma$ on $\mathbb{T}^{n} \times M$, then $\tilde{\rho}$ is also locally rigid in the analytic category.

Zeghib also proves a number of other interesting results for both volume preserving and non-volume-preserving actions and it is clear that his method has applications not stated in his note. The key point for all of his arguments is a theorem of Ghys and Cairns that says that one can linearize an analytic action of a higher rank lattice in a neighborhood of a fixed point. Zeghib proves his results by using results of Stowe [St1; St2] to find fixed points for the perturbed action and then studying the largest possible set to which the linearization around this point can be extended. We end this section by stating the theorem of Cairns and Ghys from [CGh] which Zeghib uses.

THEOREM 4.14. Let $G$ be a semisimple Lie group of real rank at least two with no compact factors and finite center and let $\Gamma<G$ be a lattice. Then every analytic action of $\Gamma$ with a fixed point $p$ is analytically linearizable in a neighborhood of $p$.

REMARKS. (1) By analytically linearizable in a neighborhood of $p$, we mean that there exists a neighborhood $U$ of $p$ and an analytic diffeomorphism $\phi$ of $U$ into the ambient manifold $M$ such that the action of $\Gamma$, conjugated by $\phi$ is the restriction of a linear action to $\phi(U)$.

(2) In the same paper, Ghys and Cairns give an example of a $C^{\infty}$ action of $S L(3, \mathbb{Z})$ on $\mathbb{R}^{8}$ fixing the origin, which is not $C^{0}$ linearizable in any neighborhood of the origin. So the assumption of analyticity in the theorem is necessary.

4.2. Actions on boundaries. In this subsection we discuss rigidity results for groups acting on homogeneous spaces known as "boundaries". In contrast to the last section, the actions we describe here never preserve a volume form, or even 
a Borel measure. We will not discuss here all the geometric, function theoretic or probabalistic reasons why these spaces are termed boundaries, but merely describe examples. For us, if $G$ is a semisimple Lie group, then a boundary of $G$ is a space of the form $G / P$ where $P$ is a connected Lie subgroup of $G$ such that the quotient $G / P$ is compact. The groups $P$ having this property are often called parabolic subgroups. The space $G / P$ is also considered a boundary for any lattice $\Gamma<G$. For more precise motivation for this terminology, see [Fu1; Fu2; Mo1; Ma4].

The simplest example of a boundary is for the group $S L(2, \mathbb{R})$ in which case the only choice of $P$ resulting in a nontrivial boundary is the group of upper (or lower) triangular matrices. The resulting quotient is naturally diffeomorphic to the circle and $S L(2, \mathbb{R})$ acts on this circle by the action on rays through the origin in $\mathbb{R}^{2}$. We can restrict this $S L(2, \mathbb{R})$ action to any lattice $\Gamma$ in $S L(2, \mathbb{R})$. The following remarkable theorem was first proved by Ghys in [Gh1]:

THEOREM 4.15. Let $\Gamma<S L(2, \mathbb{R})$ be a cocompact lattice and let $\rho$ be the action of $\Gamma$ on $S^{1}$ described above. If $\rho^{\prime}$ is any perturbation of $\rho$, then $\rho^{\prime}$ is smoothly conjugate to an action defined by another embedding $\pi^{\prime}$ of $\Gamma$ in $S L(2, \mathbb{R})$ close to the original embedding. In particular $\pi^{\prime}(\Gamma)$ is a cocompact lattice in $S L(2, \mathbb{R})$.

Ghys gives two proofs of this fact, one in [Gh1] and another different one in [Gh2]. A third and also different proof is in later work of Kononenko and Yue [KY]. Ghys' first proof derives from a remarkable global rigidity result for actions on certain three dimensional manifolds by the affine group of the line, while his second derives from rigidity results concerning certain Anosov flows on three dimensional manifolds. We remark that the fact that $\rho^{\prime}$ is continuously conjugate to an action defined by a nearby embedding into $S L(2, \mathbb{R})$ was known and so Theorem 4.15 can be viewed as a regularity theorem though this is not how the proof proceeds.

Both of Ghys' proofs pass through a statement concerning local rigidity of foliations. This uses the following variant on the construction of the induced action. Let $\Gamma$ be a cocompact lattice in $S L(2, \mathbb{R})$, and let $\rho$ be the $\Gamma$ action on $S^{1}$ defined by the action of $S L(2, \mathbb{R})$ there. There is also a $\Gamma$ action on the hyperbolic plane $\mathbb{H}^{2}$. We form the manifold $\left(\mathbb{H}^{2} \times S^{1}\right) / \Gamma_{\rho}$, where $\Gamma$ acts diagonally. This manifold is diffeomorphic to the unit tangent bundle of $\mathbb{H}^{2} / \Gamma$ which is also diffeomorphic to $S L(2, \mathbb{R}) / \Gamma$, and the foliation by planes of the form $\mathbb{H}^{2} \times\{$ point $\}$ is the weak stable foliation for the geodesic flow and also the orbit foliation for the action of the affine group. Given a $C^{r}$ perturbation $\rho^{\prime}$ of the $\Gamma$ action on $S^{1}$, we can form the corresponding bundle $\left(\mathbb{M}^{2} \times S^{1}\right) \rho^{\prime} / \Gamma_{\rho^{\prime}}$, and the foliation by planes of the form $\mathbb{H}^{2} \times\{$ point $\}$ is $C^{r}$ close to analogous foliation in $\left(\mathbb{M}^{2} \times S^{1}\right) \Gamma_{\rho}$. To show that $\rho$ and $\rho^{\prime}$ are conjugate, it suffices to find a 
diffeomorphism of $\left(\mathbb{M}^{2} \times S^{1}\right) / \Gamma_{\rho}$ conjugating the two foliations. Both of Ghys' proofs proceed by constructing such a conjugacy of foliations. This reduction to studying local rigidity of foliations has further applications in slightly different settings, see Theorems 4.16 and 4.17 below.

In later work, Ghys proved a remarkable result which characterized an entire connected component of the space of actions of $\Gamma$ on $S^{1}$. Let $X$ be the component of $\operatorname{Hom}\left(\Gamma, \operatorname{Diff}\left(S^{1}\right)\right)$ containing the actions described in Theorem 4.15. In [Gh3], Ghys showed that this component consisted entirely of actions conjugate to actions defined by embeddings $\pi^{\prime}$ of $\Gamma$ into $S L(2, \mathbb{R})$ where $\pi^{\prime}(\Gamma)$ is a cocompact lattice. This result builds on earlier work of Ghys where a similar result was proven concerning $\operatorname{Hom}\left(\Gamma, \operatorname{Homeo}\left(S^{1}\right)\right)$. A key ingredient is the use of the Euler class of the action, viewed as a bounded cocycle.

As mentioned above, Ghys' method of reducing local rigidity of an action to local rigidity of a foliation has had two more applications. The first of these is due to Katok and Spatzier [KS2].

THEOREM 4.16. Let $G$ be a semisimple Lie group with no compact factors and real rank at least two. Let $\Gamma<G$ be a cocompact lattice and $B=G / P$ a boundary for $\Gamma$. Then the $\Gamma$ action on $G / P$ is locally rigid.

The proof of this result uses an argument similar to Ghys' to reduce to a need to study regularity of foliations for perturbations of the action of certain connected abelian subgroups of $G$ on $G / \Gamma$. The result used in the proof here is the same as the one used in the proof of Theorem 4.7. For $\Gamma$ a lattice but not cocompact, some partial results are obtained by Yaskolko in his Ph.D. thesis [Yk].

Following a similar outline, Kanai proved the following:

THEOREM 4.17. Let $G=S O(n, 1)$ and $\Gamma<G$ be a cocompact lattice. Then the action of $\Gamma$ on the boundary $G / P$ is locally rigid.

Partial results in this direction were proven earlier by Chengbo Yue. Yue also proves partial results in the case where $S O(1, n)$ is replaced by any rank 1 noncompact simple Lie group.

In somewhat earlier work, Kanai had also proven a special case of Theorem 4.16. More precisely:

THEOREM 4.18. Let $\Gamma<S L(n, \mathbb{R})$ be a cocompact lattice where $n \geq 21$ and let $\rho$ be the $\Gamma$ action on $S^{n-1}$ by acting on the space of rays in $\mathbb{R}^{n}$. Then $\rho$ is locally rigid.

Kanai's proof proceeds in two steps. In the first step, he uses Thomas' notion of a projective connection to reduce the question to one concerning vanishing of certain cohomology groups. In the second step, he uses stochastic calculus to prove a vanishing theorem for the relevant cohomology groups. The first 
step is rather special, and is what restricts Kanai's attention to spheres, rather than other boundaries, which are Grassmanians. The method in the second step seems a good deal more general and should have further applications, perhaps in the context of Theorem 5.12 below. While the approach here is similar in spirit to the work of Benveniste in [Be1], it should be noted that Kanai does not use a hard implicit function theorem.

We end this subsection by recalling a construction due to Stuck, which shows that much less rigidity should be expected from actions which do not preserve volume [Sk]. Let $G$ be a semisimple Lie group and $P<G$ a minimal parabolic. Then there always exists a homomorphism $\pi: P \rightarrow \mathbb{R}$. Given any manifold $M$ and any action $s$ of $\mathbb{R}$ on $M$, we can then form the induced $G$ action $\rho_{s}$ on $(G \times M) / P$ where $P$ acts on $G$ on the left and on $M$ by $\pi \circ s$. Varying the action $s$ varies the action $\rho_{s}$. It is easy to see that if $\rho_{s}$ and $\rho_{s^{\prime}}$ are two such actions, then they are conjugate as $G$ actions if and only if $s$ and $s^{\prime}$ are conjugate. If one picks an irreducible lattice $\Gamma$ in $G \times G$, project $\Gamma$ to $G$ and restricts the actions to $\Gamma$, then it is also easy to see that the restriction of $\rho_{s}$ and $\rho_{s^{\prime}}$ to $\Gamma$ are conjugate if and only if $s$ and $s^{\prime}$ are conjugate. The author does not know a proof that this is also true if one simply takes a lattice $\Gamma$ in $G$, but believes that this is also true and may even be known.

4.3. "Flexible" actions of rigid groups. In this subsection, I discuss a sequence of results concerning flexible actions of large groups. More or less, the sequence of examples provides counterexamples to most naive conjectures of the form "all of actions of some lattice $\Gamma$ are locally rigid." There are some groups, for example compact groups and finite groups, all of whose smooth actions are locally rigid. It seems likely that there should be infinite discrete groups with this property as well, but the constructions in this subsection show that one must look beyond lattices in Lie groups for examples.

Essentially all of the examples given here derive from the simple construction of "blowing up" a point or a closed orbit, which was introduced to this subject in [KL2]. The further developments after that result are all further elaborations on one basic construction. The idea is to use the "blow up" construction to introduce distinguished closed invariant sets which can be varied in some manner to produce deformations of the action. The "blow up" construction is a classical tool from algebraic geometry which takes a manifold $N$ and a point $p$ and constructs from it a new manifold $N^{\prime}$ by replacing $p$ by the space of directions at $p$. Let $\mathbb{R} P^{l}$ be the $l$ dimensional projective space. To blow up a point, we take the product of $N \times \mathbb{R} P^{\operatorname{dim}(N)}$ and then find a submanifold where the projection to $N$ is a diffeomorphism off of $p$ and the fiber of the projection over $p$ is $\mathbb{R} P^{\operatorname{dim}(N)}$. For detailed discussion of this construction we refer the reader to any reasonable book on algebraic geometry. 
The easiest example to consider is to take the action of $S L(n, \mathbb{Z})$, or any subgroup $\Gamma<S L(n, \mathbb{Z})$ on the torus $\mathbb{T}^{n}$ and blow up the fixed point, in this case the equivalence class of the origin in $\mathbb{R}^{n}$. Call the resulting manifold $M$. Provided $\Gamma$ is large enough, e.g. Zariski dense in $S L(n, \mathbb{R})$, this action of $\Gamma$ does not preserve the measure defined by any volume form on $M$. A clever construction introduced in [KL2] shows that one can alter the standard blowing up procedure in order to produce a one parameter family of $S L(n, \mathbb{Z})$ actions on $M$, only one of which preserves a volume form. This immediately shows that this action on $M$ admits perturbations, since it cannot be conjugate to the nearby, non-volumepreserving actions. Essentially, one constructs different differentiable structures on $M$ which are diffeomorphic but not equivariantly diffeomorphic.

After noticing this construction, one can proceed to build more complicated examples by passing to a subgroup of finite index, and then blowing up several fixed points. One can also glue together the "blown up" fixed points to obtain an action on a manifold with more complicated topology. See [KL2; FW] for discussion of the topological complications one can introduce.

In [Be2] it is observed that a similar construction can be used for the action of a simple group $G$ by left translations on a homogeneous space $H / \Lambda$ where $H$ is a Lie group containing $G$ and $\Lambda<H$ is a cocompact lattice. Here we use a slightly more involved construction from algebraic geometry, and "blow up" the directions normal to a closed submanifold. I.e. we replace some closed submanifold $N$ in $H / \Lambda$ by the projectived normal bundle to $N$. In all cases we consider here, this normal bundle is trivial and so is just $N \times \mathbb{R} P^{l}$ where $l=\operatorname{dim}(H)-\operatorname{dim}(N)$.

Benveniste used his construction to produce more interesting perturbations of actions of higher rank simple Lie group $G$ or a lattice $\Gamma$ in $G$. In particular, he produced volume preserving actions which admit volume preserving perturbations. He does this by choosing $G<H$ such that not only are there closed $G$ orbits but so that the centralizer $Z=Z_{H}(G)$ of $G$ in $H$ has no-trivial connected component. If we take a closed $G$ orbit $N$, then any translate $z N$ for $z$ in $Z$ is also closed and so we have a continuum of closed $G$ orbits. Benveniste shows that if we choose two closed orbits $N$ and $z N$ to blow up and glue, and then vary $z$ in a small open set, the resulting actions can only be conjugate for a countable set of choices of $z$.

This construction is further elaborated in [F1]. Benveniste's construction is not optimal in several senses, nor is his proof of rigidity. In [F1], I give a modification of the construction that produces nonconjugate actions for every choice of $z$ in a small enough neighborhood. By blowing up and gluing more pairs of closed orbits, this allows me to produce actions where the space of deformations contains a submanifold of arbitrarily high, finite dimension. Fur- 
ther, Benveniste's proof that the deformation are nontrivial is quite involved and applies only to higher rank groups. In [F1], I give a different proof of nontriviality of the deformations, using consequences of Ratner's theorem to due Witte and Shah [R; Sh; W2]. This shows that the construction produces nontrivial perturbations for any semisimple $G$ and any lattice $\Gamma$ in $G$.

In $[B F]$ we show that none of these actions preserve any rigid geometric structure in the sense of Gromov. It is possible that any action of a higher rank lattice which preserves a rigid geometric structure is locally rigid. It is also possible that any such action is generalized quasiaffine.

4.4. Infinitesimal rigidity. In [Z4], Zimmer introduced a notion of infinitesimal rigidity motivated by Weil's Theorem 3.2 and the analogy between finite dimensional Lie algebras and vector fields. Let $\rho$ be a smooth action of a group $\Gamma$ on a manifold $M$, then $\rho$ is infinitesimally rigid if $H^{1}\left(\Gamma\right.$, $\left.\operatorname{Vect}^{\infty}(M)\right)=0$. Here the $\Gamma$ action on $\operatorname{Vect}^{\infty}(M)$ is given by the derivative of $\rho$. The notion of infinitesimal rigidity was introduced with the hope that one could prove an analogue of Weil's Theorem 3.2 and then results concerning infinitesimal rigidity would imply results concerning local rigidity. Many infinitesimal rigidity results were then proven, see [H2; Ko; L; LZ; Q3; Z6]. For some more results on infinitesimal rigidity, see Theorems 5.10 and 5.11. Also see subsection 5.2 for a discussion of known results on the relation between infinitesimal and local rigidity.

\section{Recent developments}

In this section we discuss the most recent dramatic developments in the field. The first subsection discusses work of the author and Margulis on rigidity of actions of higher rank groups and lattices. Our main result is that if $H$ is the real points of an algebraic group defined over $\mathbb{R}$ and $\Lambda<H$ is a cocompact lattice, then any affine action of $G$ or $\Gamma$ on $H / \Lambda$ is locally rigid. This work is quite involved and spans a sequence of three long papers [FM1; FM2; FM3]. One of the main goals of subsection 5.1 is to provide something of a "reader's guide" to those papers.

The second subsection discusses some recent developments involving more geometric and analytic approaches to local rigidity. Till this point, the study of local rigidity of group actions has been dominated by algebraic ideas and hyperbolic dynamics with the exception of [Ka1] and [Be2]. The results described in subsection 5.2 represent (the beginning of) a dramatic development in analytic and geometric techniques. The first of these is the work of Damjanovich and Katok on local rigidity of certain partially hyperbolic affine actions of abelian groups on tori using a KAM approach [DK1; DK2]. The second is the author's 
proof of a criterion for local rigidity of groups actions modelled on Weil's Theorem 3.2 and proven using Hamilton's implicit function theorem [F2]. This result currently has an unfortunate "side condition" on second cohomology that makes it difficult to apply.

The final subsection concerns a few other very recent results and developments that the author feels point towards the future development of the field.

5.1. The work of Margulis and the author. Let $G$ be a (connected) semisimple Lie group with all simple factors of real rank at least two, and $\Gamma<G$ is a lattice. The main result of the papers [FM1; FM2; FM3] is:

THEOREM 5.1. Let $\rho$ be a volume preserving quasiaffine action of $G$ or $\Gamma$ on a compact manifold $X$. Then the action locally rigid.

REMARKS. (1) This result subsumes essentially all of the theorems in subsection 4.1, excepting those concerning actions of abelian groups.

(2) In [FM3] we also achieve some remarkable results for perturbations of very low regularity. In particular, we prove that any perturbation which is a $C^{3}$ close $C^{3}$ action is conjugate back to the original action by a $C^{2}$ diffeomorphism.

(3) The statement here is slightly different than that in [FM3]. Here $X=$ $H / L \times M$ with $L$ cocompact, while there $X=H / \Lambda \times M$ with $\Lambda$ discrete and cocompact. An essentially algebraic argument using results in [W1], shows that possibly after changing $H$ and $M$, these hypotheses are equivalent.

Another main result of the research resulting in Theorem 5.1 is the following:

THEOREM 5.2. Let $\Gamma$ be a discrete group with property $(T)$. Let $X$ be a compact smooth manifold, and let $\rho$ be a smooth action of $\Gamma$ on $X$ by Riemannian isometries. Then $\rho$ is locally rigid.

REMARKS. (1) A key step in the proof of Theorem 5.1 is a foliated version of Theorem 5.2.

(2) As in Theorem 5.1, there is a finite regularity version of Theorem 5.2 and it's foliated generalization, we refer the reader to [FM2] for details.

The remainder of this subsection will consist of a sketch of the proof of Theorem 5.1. The intention is essentially to provide a reader's guide to the three papers [FM1; FM2; FM3]. Throughout the remainder of this subsection, to simplify notation, we will discuss only the case of affine $\Gamma$ actions on $H / \Lambda$ with $\Lambda$ a cocompact lattice. The proof for connected groups and quasiaffine action on $X=H / \Lambda \times M$ is similar. To further simplify the discussion, we assume that $\rho^{\prime}$ is a $C^{\infty}$ perturbation of $\rho$. 
Step 1: An invariant "central" foliation for the perturbed action and leaf conjugacy. To begin the discussion and the proof, we need some knowledge of the structure of the affine actions considered. By [FM1, Theorem 6.4], there is a finite index subgroup $\Gamma^{\prime}<\Gamma$ such that the action of $\Gamma^{\prime}$ on $H / \Lambda$ is given by a homomorphism $\sigma: \Gamma^{\prime} \rightarrow \operatorname{Aut}(H) \ltimes H$. We simplify the discussion by assuming $\Gamma=\Gamma^{\prime}$ throughout. Using Margulis superrigidity theorems, which are also used in the proof of [FM1, Theorem 6.4], it is relatively easy to understand the maximal central foliation $\mathfrak{F}$ for $\rho$ : there is a subgroup $Z<H$ whose orbit foliation is exactly the central foliation. For example, if $G<H$ acts on $H / \Lambda$ by left translations and $\rho$ is restriction of that action to $\Gamma$, then $Z=Z_{H}(G)$. For details on what $Z$ is more generally, see [FM1].

Given a perturbation $\rho^{\prime}$ of $\rho$, we begin by finding a $\rho^{\prime}$ invariant foliation $\mathfrak{F}^{\prime}$ and a leaf conjugacy $\phi$ from $(H / \Lambda, \rho, \mathfrak{F})$ to $\left(H / \Lambda, \rho^{\prime}, \mathfrak{F}^{\prime}\right)$. To do this, we apply a result concerning local rigidity of cocycles over actions of higher rank groups and lattices to the Margulis-Qian cocycle defined by the perturbation. As the statements of the local rigidity results for cocycles are somewhat technical, we refer the reader to [FM1, Theorems 1.1 and 5.1]. Those Theorems are proven in Section 5 of that paper using results in Section 4 concerning orbits in representation varieties as well as the cocycle superrigidity theorems. The construction of the leaf conjugacy is completed in [FM3, Section 2.2] using [FM1, Theorem 1.8]. We remark that we actually construct $\phi^{-1}$ rather than $\phi$. The paper [FM1] also contains a proof of superrigidity for cocycles that results in many technical improvements to that result.

Step 2: Smoothness of the central foliation, reduction to a foliated perturbation. The next step in the proof is to show that $\mathfrak{F}^{\prime}$ is a foliation by smooth leaves. In fact, it is only possible to show at this point that it is a foliation by $C^{k}$ leaves for some $k$ depending on $\rho$ and $\rho^{\prime}$ and particularly on the $C^{1}$ size of the perturbation. This is done using the work of Hirsch, Pugh and Shub described in subsection 3.2. If the central foliation for $\rho$ is the central foliation for $\rho(\gamma)$ for some single element $\gamma$ in $\Gamma$, this amounts to showing that the foliation $\mathfrak{F}^{\prime}$ constructed in step one is the same foliation as the central foliation for $\rho^{\prime}(\gamma)$ constructed in the proof of Theorem 3.3. To prove this, one needs to analyze the proof of Theorem 3.3. More generally, we show, in [FM3, Section 3.2] that there is a finite collection of elements $\gamma_{1}, \ldots, \gamma_{k}$ in $\Gamma$ such that each leaf of the foliation $\mathfrak{F}$ is a transverse intersection of central leaves of $\rho\left(\gamma_{1}\right), \ldots, \rho\left(\gamma_{k}\right)$. One then needs to combine an analysis of the proof of Theorem 3.3 with some arguments concerning persistence of transversality under certain kinds of perturbations. This argument is carried out in [FM3, Section 3.3].

Once we know that $\mathfrak{F}^{\prime}$ is $C^{k}$, it is easy to see that the leaf conjugacy $\phi$ is $C^{k}$ and $C^{k}$ small along leaves of $\mathfrak{F}$ though all derivatives are only continuous in the 
transverse direction. Conjugating the action $\rho^{\prime}$ by $\phi$, we obtain a new action $\rho^{\prime \prime}$ on $H / \Lambda$ which preserves $\mathfrak{F}$. This action is only continuous, but it is $C^{0}$ close to $\rho$ and $C^{k}$ and $C^{k}$ close to $\rho$ along leaves of $\mathfrak{F}$. In [FM2; FM3] we refer to perturbations of this type as foliated perturbations.

Step 3: Conjugacy along the central foliation. The next step is to apply a foliated generalization of Theorem 5.2 to the actions $\rho$ and $\rho^{\prime \prime}$. The exact result we apply is [FM2, Theorem 2.11] which produces a semiconjugacy $\psi$ between $\rho$ and $\rho^{\prime}$. This result is somewhat involved to state and the regularity of $\psi$ is hard to describe. The map $\psi$ is $C^{k-1-\varepsilon}$ along the leaves of $\mathfrak{F}$ at almost all points in $H / \Lambda$, for $\varepsilon$ depending on the size of the perturbation, but only transversely measurable. In addition, the map $\psi$ satisfies a certain Sobolev estimate, that implies that it is $C^{k-1-\varepsilon}$ small in a small ball in $\mathfrak{F}$ at most points, and that the $C^{k-1-\varepsilon}$ norm is only large on very small sets. Rather than try to make this precise here, we include a sketch of the proof of Theorem 5.2. Before doing so we remark that the map $\varphi=\phi \circ \psi$ is a semiconjugacy from between the $\Gamma$ action $\rho$ and the $\Gamma$ action $\rho^{\prime}$. The last step in the argument is to improve the regularity of $\varphi$ which we will discuss following the sketch of the proof of Theorem 5.2

We recall two definitions and another theorem from [FM2].

DEFINITION 5.3. Let $\varepsilon \geq 0$ and $Z$ and $Y$ be metric spaces. Then a map $h: Z \rightarrow Y$ is an $\varepsilon$-almost isometry if

$$
(1-\varepsilon) d_{Z}(x, y) \leq d_{Y}(h(x), h(y)) \leq(1+\varepsilon) d_{Z}(x, y)
$$

for all $x, y \in Z$.

The reader should note that an $\varepsilon$-almost isometry is a bilipschitz map. We prefer this notation and vocabulary since it emphasizes the relationship to isometries.

Definition 5.4. Given a group $\Gamma$ acting on a metric space $X$, a compact subset $S$ of $\Gamma$ and a point $x \in X$. The number $\sup _{k \in S} d(x, k \cdot x)$ is called the $S$-displacement of $x$ and is denoted $\operatorname{disp}_{S}(x)$.

THEOREM 5.5. Let $\Gamma$ be a locally compact, $\sigma$-compact group with property $(T)$ and $S$ a compact generating set. There exist positive constants $\varepsilon$ and $D$, depending only on $\Gamma$ and $S$, such that for any continuous action of $\Gamma$ on a Hilbert space $\mathscr{H}$ where $S$ acts by $\varepsilon$-almost isometries there is a fixed point $x$; furthermore for any $y$ in $X$, the distance from $y$ to the fixed set is not more than $D \operatorname{disp}_{S}(y)$.

We now sketch the proof of Theorem 5.2 for Theorem 5.5. Given a compact Riemannian manifold $X$, there is a canonical construction of a Sobolev inner product on $C^{k}(X)$ such that the Sobolev inner product is invariant under isometries of the Riemannian metric, see [FM2, Section 4]. We call the completion 
of $C^{k}(X)$ with respect to the metric induced by the Sobolev structure $L^{2, k}(X)$. Given an isometric $\Gamma$ action $\rho$ on a manifold $M$ there may be no nonconstant $\Gamma$ invariant functions in $L^{2, k}(X)$. However, if we pass to the diagonal $\Gamma$ action on $X \times X$, then any function of the distance to the diagonal is $\Gamma$ invariant and, if $C^{k}$, is in $L^{2, k}(X \times X)$.

We choose a smooth function $f$ of the distance to the diagonal in $X \times X$ which has a unique global minimum at $x$ on $\{x\} \times X$ for each $x$, and such that any function $C^{2}$ close to $f$ also has a unique minimum on each $\{x\} \times X$. This is guaranteed by a condition on the Hessian and the function is obtained from $d(x, y)^{2}$ by renormalizing and smoothing the function away from the diagonal. This implies $f$ is invariant under the diagonal $\Gamma$ action defined by $\rho$. Let $\rho^{\prime}$ be another action $C^{k}$ close to $\rho$. We define a $\Gamma$ action on $X \times X$ by acting on the first factor by $\rho$ and on the second factor by $\rho^{\prime}$. For the resulting action $\bar{\rho}^{\prime}$ of $\Gamma$ on $L^{2, k}(X \times X)$ and every $\gamma \in S$, we show that $\bar{\rho}^{\prime}(k)$ is an $\varepsilon$-almost isometry and that the $S$-displacement of $f$ is a small number $\delta$, where both $\varepsilon$ and $\delta$ can be made arbitrarily small by choosing $\rho^{\prime}$ close enough to $\rho$. Theorem 5.5 produces a $\bar{\rho}^{\prime}$ invariant function $f^{\prime}$ close to $f$ in the $L^{2, k}$ topology. Then $f^{\prime}$ is $C^{k-\operatorname{dim}(X)}$ close to $f$ by the Sobolev embedding theorems and if $k-\operatorname{dim}(X) \geq 2$, then $f$ has a unique minimum on each fiber $\{x\} \times X$ which is close to $(x, x)$. We verify that the set of minima is a $C^{k-\operatorname{dim}(X)-1}$ submanifold and, in fact, the graph of a conjugacy between the $\Gamma$ actions on $X$ defined by $\rho$ and $\rho^{\prime}$.

Note that this argument yields worse regularity than we discussed in the foliated context or than is stated in Theorem 5.2. There are considerable difficulties involved in achieving lower loss of regularity or in producing a $C^{\infty}$ conjugacy and we do not dwell on these here, but refer the reader to [FM2] and also to some discussion in the next step.

Step 4: Regularity of the conjugacy. We improve the regularity of $\varphi$ in three stages. First we show it is a homeomorphism in [FM3, Section 5.2]. The key to this argument is proving that there is a finite collection $\gamma_{1}, \ldots, \gamma_{k}$ of elements of $\Gamma$ such that $\varphi$ takes stable foliations for $\rho\left(\gamma_{i}\right)$ to stable foliations for $\rho^{\prime}\left(\gamma_{i}\right)$. If $\varphi$ were continuous, this is both easy and classical. In our context, we require a density point argument to prove this, which is given in [FM3, Section 5.1]. Once we have that stable foliations go to stable foliations, we use this to show that $\varphi$ is actually uniformly continuous along central foliations and then patch together continuity along various foliations. (In [FM3, Section 5.3], we show how to remove the assumption, made above, that the $\Gamma$ action lifts from $H / \Lambda$ to $H$. This cannot be done until we have produced a continuous conjugacy.)

The next stage is to show that $\varphi$ is a finite regularity diffeomorphism. To show this, we show that $\varphi$ is smooth (with estimates) along certain foliations which span the tangent space. This step is essentially an implementation of the method 
of Katok and Spatzier described in subsection 4.1. A few technical difficulties occur as we need to keep careful track of estimates in the method for use at later steps and because we need to identify ergodic components of the measure for certain elements in the unperturbed action. After applying the Katok-Spatzier method, we have that $\varphi$ is smooth along many contracting directions and smooth along the central foliation, and then use a fairly standard argument involving elliptic operators to show that it is actually smooth, and even $C^{k^{\prime}}$ small, for $k^{\prime}=k-1-\varepsilon-\frac{1}{2} \operatorname{dim}(H)$. It would be interesting to see if one could lose less regularity at this step, for example by a method like Journé's. A key difficulty in adapting the method of [Jn] is that we only have a Sobolev estimate along central leaves and not a uniform one.

The last stage of the argument is to show that $\varphi$ is smooth. There are two parts to this argument. The first is to show that if $\rho$ and $\rho^{\prime}$ are $C^{k}$ close, we can actually show that $\varphi$ is $C^{l}$ for some $l \geq k$. The main difficulty here is obtaining better regularity in the foliated version of Theorem 5.2. This requires the use of estimates on convexity of derivatives and estimates on compositions of diffeomorphisms, as well as an iterative method of constructing the semiconjugacy $\psi$, for this we refer the reader to [FM2, Sections 6 and 7.3]. Once we know we can produce a conjugacy of greater regularity, we can then approximate $\varphi$ in the $C^{l}$ topology by a $C^{\infty}$ map and smoothly conjugate $\rho^{\prime}$ to a very small $C^{l}$ perturbation of $\rho$. The point is to iterate this procedure while obtaining estimates on the size of the conjugacy produced at each step. We then show that the iteration converges to produce a smooth conjugacy. We give here a general theorem whose proof follows from arguments in [FM2; FM3].

It is convenient to fix right invariant metrics $d_{l}$ on the connected components of $\operatorname{Diff}^{l}(X)$ with the additional property that if $\varphi$ is in the connected component of $\operatorname{Diff}{ }^{\infty}(X)$, then $d_{l}(\varphi$, Id $) \leq d_{l+1}(\varphi$, Id $)$. To fix $d_{l}$, it suffices to define inner products $\langle,\rangle_{l}$ on $\operatorname{Vect}^{l}(X)$ which satisfy $\langle V, V\rangle_{l} \leq\langle V, V\rangle_{l+1}$ for $V \in \operatorname{Vect}^{\infty}(X)$. As remarked in [FM2, Section 6], after fixing a Riemannian metric $g$ on $X$, it is straightforward to introduce such metrics using the methods of [FM2, Section 4].

DEFINITION 5.6. Let $\Gamma$ be a group, $M$ a compact manifold and assume $\rho$ : $\Gamma \rightarrow \operatorname{Diff}^{\infty}(M)$. We say $\rho$ is strongly $C^{k, l, n}$ locally rigid if for every $\varepsilon>0$ there exists $\delta>0$ such that if $\rho^{\prime}$ is an action of $\Gamma$ on $M$ with $d_{k}\left(\rho^{\prime}(g) \rho(g)^{-1}\right.$, Id $)<\delta$ for all $g \in K$ then there exists a $C^{l}$ conjugacy $\varphi$ between $\rho$ and $\rho^{\prime}$ such that $d_{k-n}(\varphi, \mathrm{Id})<\varepsilon$.

We are mainly interested in the case where $l>k$.

THEOREM 5.7. Let $\Gamma$ be a group, $M$ a compact manifold and assume $\rho$ : $\Gamma \rightarrow \operatorname{Diff}^{\infty}(M)$. Assume that there are constants $n>0$ and $k_{0} \geq 0$ and that for 
every $k>k_{0}$ there exists an $l>k$ such that $\rho$ is strongly $C^{k, l, n}$ locally rigid. Then $\rho$ is $C^{\infty}$ locally rigid.

The proof of Theorem 5.7 follows [FM3, Corollary 7.2], though the result is not stated in this generality there.

\subsection{KAM, implicit function theorems: work of Damjanovich-Katok and}

the author. In this subsection, we discuss some new results that use more geometric/analytic methods to approach questions of local rigidity. These methods are entirely independent of methods using hyperbolic dynamics and appear likely to be robustly applicable.

We begin with a theorem of Katok and Damjanovich concerning abelian groups of toral automorphisms. Here we consider actions $\pi: \mathbb{Z}^{n} \rightarrow \operatorname{Diff}^{\infty}\left(\mathbb{T}^{m}\right)$ where $\pi\left(\mathbb{Z}^{n}\right)$ lies in $G L(m, Z)$ acting on $\mathbb{T}^{m}$ by linear automorphisms or more generally where $\pi\left(\mathbb{Z}^{n}\right)$ acts affinely on $\mathbb{T}^{m}$. An affine factor $\pi^{\prime}$ of $\pi$ is another affine action $\pi^{\prime}: \mathbb{Z}^{n} \rightarrow \mathbb{T}^{l}$ and there is an affine map $\phi: \mathbb{T}^{n} \rightarrow \mathbb{T}^{l}$ such that $\phi \circ \pi(v)=\pi^{\prime}(v) \circ \phi$ for every $v \in \mathbb{Z}^{n}$. We say a factor $\pi^{\prime}$ has rank one if $\pi^{\prime}\left(\mathbb{Z}^{n}\right)$ has a finite index cyclic subgroup.

THEOREM 5.8. Let $\pi: \mathbb{Z}^{n} \rightarrow \operatorname{Aff}\left(\mathbb{T}^{m}\right)$ have no rank one factors. Then $\pi$ is locally rigid.

This theorem is proven by a KAM method. One should note that all the theorems on actions of abelian groups by toral automorphisms stated in subsection 4.1 are special cases of this theorem. (This is not quite literally true. Those theorems apply to perturbations that are only $C^{1}$ close, while the result currently under discussion only applies to actions that are close to very high order.) It is also worthwhile to note that Theorem 5.8 can be proven using no techniques of hyperbolic dynamics.

We now briefly describe the KAM method. Let $\Gamma$ be a finitely generated group and $\pi: \Gamma \rightarrow \operatorname{Diff}^{\infty}(M)$ a homomorphism. To apply a KAM-type argument, define $L: \operatorname{Diff}^{\infty}(M)^{k} \times \operatorname{Diff}^{\infty}(M) \rightarrow \operatorname{Diff}^{\infty}(M)^{k}$ by taking

$$
L\left(\phi_{1}, \ldots, \phi_{k}, f\right)=\left(\pi\left(\gamma_{1}\right) \circ f \circ \phi_{1} \circ f^{-1}, \ldots, \pi\left(\gamma_{1}\right) \circ f \circ \phi_{1} \circ f^{-1}\right)
$$

where $\pi: \Gamma \rightarrow \operatorname{Diff}^{\infty}(M)$ is a homomorphism. If $\pi^{\prime}$ is another $\Gamma$ action on $M$ then $L\left(\pi^{\prime}\left(\gamma_{1}\right), \ldots, \pi^{\prime}\left(\gamma_{k}\right), f\right)=(\mathrm{Id}, \ldots, \mathrm{Id})$ implies $f$ is a conjugacy between $\pi$ and $\pi^{\prime}$, so the problem of finding a conjugacy is the same as finding a diffeomorphism $f$ which solves $L\left(\pi^{\prime}\left(\gamma_{1}\right), \ldots, \pi^{\prime}\left(\gamma_{k}\right), f\right)=(\mathrm{Id}, \ldots$, Id $)$ subject to the constraint that $\pi^{\prime}$ is a $\Gamma$ action.

The $K A M$ method proceeds by taking the derivative $D L$ of $L$ at $(\pi, f)$ and solving the resulting linear equation instead subject to a linear constraint that is the derivative of the condition that $\pi^{\prime}$ is a $\Gamma$ action. This produces an "approximate solution" to the nonlinear problem and one proceeds by an iteration. If the 
original perturbation is of size $\varepsilon$ then the perturbation obtained after one step in the iteration is of size $\varepsilon^{2}$ at least to whatever order one can control the size of the solution of $D L$. This allows one to show that the iteration converges even under conditions where there is some dramatic "loss of regularity", i.e. when solutions of $D L$ are only small to much lower order than the initial data. A standard technique used to combat this loss of regularity is to alter the equation given by $D L$ by inserting smoothing operators. That one can solve the linearized equation modified by smoothing operators in place of the original linearized equation and still expect to prove convergence of the iterative procedure depends heavily on the quadratic convergence just described. The main difficulty in applying this outline is obtaining so-called tame estimates on inverses of linearized operators. For a definition of a tame estimate, see following Theorem 5.12.

The KAM method is often presented as a method for proving hard implicit function theorems. The paradigmatic theorem of this kind is due to Hamilton [Ha1; Ha2], and is used by the author in the proof of the following theorem. For a brief discussion of the relation between this work and that of Katok and Damjanovich, see the end of this subsection.

THEOREM 5.9. Let $\Gamma$ be a finitely presented group, $(M, g)$ a compact Riemannian manifold and $\pi: \Gamma \rightarrow \operatorname{Isom}(M, g) \subset \operatorname{Diff}^{\infty}(M)$ a homomorphism. If $H^{1}\left(\Gamma\right.$, $\left.\operatorname{Vect}^{\infty}(M)\right)=0$ and $H^{2}\left(\Gamma\right.$, Vect $\left.{ }^{\infty}(M)\right)$ is Hausdorff in the tame topology, the homomorphism $\pi$ is locally rigid as a homomorphism into $\operatorname{Diff}^{\infty}(M)$.

I believe the condition on $H^{2}\left(\Gamma\right.$, Vect $\left.{ }^{\infty}(M)\right)$ should hold automatically under the other hypotheses of the theorem. If this is true, then one has a new proof of Theorem 5.2 using a result in [LZ]. There are some other infinitesimal rigidity results that would then yield more novel applications. For example:

THEOREM 5.10. Let $\Gamma$ be an irreducible lattice in a semisimple Lie group $G$ with real rank at least two. Then for any Riemannian isometric action of $\Gamma$ on a compact manifold $H^{1}\left(\Gamma\right.$, Vect $\left.{ }^{\infty}(M)\right)=0$.

Theorem 5.10 naturally applies in greater generality, in particular to irreducible $S$-arithmetic lattices and to irreducible lattices in products of more general locally compact groups.

To give another result on infinitesimal rigidity, we require a definition. For certain cocompact arithmetic lattices $\Gamma$ in a simple group $G$, the arithmetic structure of $\Gamma$ comes from a realization of $\Gamma$ as the integer points in $G \times K$ where $K$ is a compact Lie group. In this case it always true that the projection to $G$ is a lattice and the projection to $K$ is dense. We say a $\Gamma$ action is arithmetic isometric if it is defined by projecting $\Gamma$ to $K$, letting $K$ act by $C^{\infty}$ diffeomorphisms on a compact manifold $M$ and restricting the $K$ action to $\Gamma$. 
THEOREM 5.11. For certain congruence lattices $\Gamma<S U(1, n)$, any arithmetic isometric action of $\Gamma$ has $H^{1}\left(\Gamma\right.$, $\left.\operatorname{Vect}^{\infty}(M)\right)=0$.

For a description of which lattices this applies to, we refer the reader to [F2]. The theorem depends on deep results of Clozel [Cl].

Theorem 5.9 actually follows from the following, more general result.

THEOREM 5.12. Let $\Gamma$ be a finitely presented group, $M$ a compact manifold, and $\pi: \Gamma \rightarrow \operatorname{Diff}^{\infty}(M)$ a homomorphism. If $H^{1}\left(\Gamma, \operatorname{Vect}^{\infty}(M)\right)=0$ and the sequence

$$
C^{0}\left(\Gamma, \operatorname{Vect}^{\infty}(M)\right) \stackrel{d_{1}}{\longrightarrow} C^{1}\left(\Gamma, \operatorname{Vect}^{\infty}(M)\right) \stackrel{d_{2}}{\longrightarrow} C^{2}\left(\Gamma, \operatorname{Vect}^{\infty}(M)\right)
$$

admits a tame splitting then the homomorphism $\pi$ is locally rigid. I.e. $\pi$ is locally rigid provided there exist tame linear maps

$$
V_{1}: C^{1}\left(\Gamma, \operatorname{Vect}^{\infty}(M)\right) \rightarrow C^{0}\left(\Gamma, \operatorname{Vect}^{\infty}(M)\right)
$$

and

$$
V_{2}: C^{2}\left(\Gamma, \operatorname{Vect}^{\infty}(M)\right) \rightarrow C^{1}\left(\Gamma, \operatorname{Vect}^{\infty}(M)\right)
$$

such that $d_{1} \circ V_{1}+V_{2} \circ d_{2}$ is the identity on $C^{1}\left(\Gamma\right.$, $\left.\operatorname{Vect}^{\infty}(M)\right)$.

Here $C^{i}\left(\Gamma, \operatorname{Vect}^{\infty}\right)$ is the group of $i$-cochains with values in $\operatorname{Vect}{ }^{\infty}(M)$ and $d_{i}$ are the standard coboundary maps where we have identified the cohomology of $\Gamma$ with the cohomology of a $K(\Gamma, 1)$ space with one vertex, one edge for each generator in our presentation of $\Gamma$ and one 2 cell for each relator in our presentation of $\Gamma$. A map $L$ is called tame if there is an estimate of the type $\|L v\|_{k} \leq C_{k}\|v\|_{k+r}$ for a fixed choice of $r$. Here the $\|\cdot\|_{l}$ can be taken to be the $C^{l}$ norm on cochains with values in $\operatorname{Vect}^{\infty}(M)$. This notion clearly formalizes the notion of being able to solve an equation with some loss of regularity.

The proof of Theorem 5.12 proceeds by reducing the question to Hamilton's implicit function theorem for short exact sequences and is similar in outline to Weil's proof of Theorem 3.2.

Fix a finitely presented group $\Gamma$ and a presentation of $\Gamma$. This is a finite collection $S$ of generators $\gamma_{1}, \ldots, \gamma_{k}$ and finite collection $R$ of relators $w_{1}, \ldots, w_{r}$ where each $w_{i}$ is a finite word in the $\gamma_{j}$ and their inverses. More formally, we can view each $w_{i}$ as a word in an alphabet on $k$ letters. Let $\pi: \Gamma \rightarrow \operatorname{Diff}^{\infty}(M)$ be a homomorphism, which we can identify with a point in $\operatorname{Diff}^{\infty}(M)^{k}$ by taking the images of the generators. We have a complex:

$$
\operatorname{Diff}^{\infty}(M) \stackrel{P}{\longrightarrow} \operatorname{Diff}^{\infty}(M)^{k} \stackrel{Q}{\longrightarrow} \operatorname{Diff}^{\infty}(M)^{r}
$$

Where $P$ is defined by taking $\psi$ to $\left(\psi \pi\left(\gamma_{1}\right) \psi^{-1}, \ldots, \psi \pi\left(\gamma_{k}\right) \psi^{-1}\right)$ and $Q$ is defined by viewing each $w_{i}$ as a word in $k$ letters and taking $\left(\psi_{1}, \ldots, \psi_{k}\right)$ to 
$\left(w_{1}\left(\psi_{1}, \ldots, \psi_{k}\right), \ldots, w_{r}\left(\psi_{1}, \ldots, \psi_{k}\right)\right)$. To this point this is simply Weil's proof where $\operatorname{Diff}^{\infty}(M)$ is replacing a finite dimensional Lie group $H$. Letting Id be the identity map on $M$, it follows that $P(\mathrm{Id})=\pi$ and $Q(\pi)=(\mathrm{Id}, \ldots, \mathrm{Id})$. Also note that $Q^{-1}\left(\operatorname{Id}_{M}, \ldots, \operatorname{Id}_{M}\right)$ is exactly the space of $\Gamma$ actions. Note that $P$ and $Q$ are $\operatorname{Diff}^{\infty}(M)$ equivariant where $\operatorname{Diff}^{\infty}(M)$ acts on itself by left translations and on $\operatorname{Diff}^{\infty}(M)^{k}$ and $\operatorname{Diff}^{\infty}(M)^{r}$ by conjugation. Combining this equivariance with Hamilton's implicit function theorem, I show that local rigidity is equivalent to producing a tame splitting of the sequence

$$
\operatorname{Vect}^{\infty}(M) \stackrel{D P_{\mathrm{Id}}}{\longrightarrow} \operatorname{Vect}^{\infty}(M)^{k} \stackrel{D Q_{\pi}}{\longrightarrow} \operatorname{Vect}^{\infty}(M)^{r}
$$

To complete the proof of Theorem 5.12 requires that one compute $D P_{\mathrm{Id}}$ and $D Q_{\pi}$ in order to relate the sequence in equation (5-2) to the cohomology sequence in Theorem 5.12.

We remark here that the information needed to split the sequence in Theorem 5.12 is quite similar to the information one would need to apply a KAM method. This is not particularly surprising as Hamilton's implicit function theorem is a formalization of the KAM method. In particular, to prove Theorem 5.8, one can apply Theorem 5.12 using estimates and constructions from [DK2, Section 3] to produce the required tame splitting. This avoids the use of the explicit KAM argument in [DK2, Section 4].

Finally, we remark that there is a theorem of Fleming that is an analogue of Theorems 5.9 and 5.12 in the setting of finite, or finite Sobolev, regularity [Fl]. This is proven using an infinite dimensional variant of Stowe's fixed point theorems, though it has recently been reproven by An and Neeb using a new implicit function theorem [AN]. With either proof, this result also has a similar condition on second cohomology. We remark here that due to the nature of the respective topologies on spaces of vector fields, the condition on second cohomology in the work of Fleming or An-Neeb is considerably stronger than what is needed in Theorems 5.9 and 5.12. As an illustration, no version of Theorem 5.8 can be proven using these results. This is because a cohomological equation can have solutions with tame estimates, i.e. with some loss of regularity, without having solutions with an estimate at any fixed regularity.

5.3. Further results. In this subsection, we describe a few more recent developments related to the results discussed so far. These results are either very recent or somewhat removed from the main stream of research.

The first result we discuss concerns actions of lattices in $\operatorname{Sp}(1, n)$ or $F_{4}^{-20}$ and is due to T. J. Hitchman. We state here only a special case of his results. For this result, we assume that $\Gamma$ is an arithmetic subgroup of $\operatorname{Sp}(1, n)$ or $F_{4}^{-20}$ in the standard $\mathbb{Q}$ structures on those groups. This means that $\Gamma$ is a finite index 
subgroup of the integer points in the standard matrix representation of these groups. This means that in the defining representation of $\operatorname{Sp}(1, n)$ or $F_{4}^{-20}$ on $\mathbb{R}^{m}$ the action of $\Gamma$ preserves the integer lattice $\mathbb{Z}^{m}$ and therefore defines an action $\rho$ of $\Gamma$ on $\mathbb{T}^{m}$.

THEOREM 5.13. The action $\rho$ defined in the last paragraph is deformation rigid.

The proof proceeds in two steps. Building a path of $C^{0}$ conjugacies follows more or less as in [H1], see subsection 4.1 above. The main novelty in [Hi] is the proof that these conjugacies are in fact smooth. Theorem 5.13 is a special case of the results obtained in [Hi].

Another recent development should lead to a common generalization of Theorems 5.8 and 4.7. For example, one can consider actions of an abelian subgroup $\mathbb{R}^{k}$ of the full diagonal group $\mathbb{R}^{n-1}$ in $S L(n, \mathbb{R})$ on $S L(n, \mathbb{R}) / \Lambda$ where $\Lambda$ is a cocompact lattice. In this context, Damjanovich and Katok are developing a more geometric approach in contrast to the analytic method of [DK1; DK2]. In [DK3], under a natural nondegeneracy condition on the subgroup $\mathbb{R}^{k}<\mathbb{R}^{n-1}$, the authors prove the cocycle rigidity result required to generalize Theorem 4.7 to the natural result for the $\mathbb{R}^{k}$ action. Here there are "additional trivial perturbations" of the action arising from $\operatorname{Hom}\left(\mathbb{R}^{k}, \mathbb{R}^{n-1}\right)$. A rigidity theorem in this context is work in progress, see [DK3] for some discussion.

To close this section, we mention two other recent works. The first is a paper by Burslem and Wilkinson which investigates local and global rigidity questions for actions of certain solvable groups on the circle. Particularly striking is their construction of group actions which admit $C^{r}$ perturbations but no $C^{r+1}$ perturbations for every integer $r$. The second is a paper by M. Einsiedler and T. Fisher in which the method of proof of Theorem 4.6 is extended to affine actions of $\mathbb{Z}^{d}$ where the matrices generating the group action have nontrivial Jordan blocks. For perturbations of the group action which are close to very high order, this result follows from Theorem 5.8, but in $[\mathrm{EF}]$ the result only requires that the perturbation be $C^{1}$ close to the original action.

\section{Directions for future research and conjectures}

In this section, I mention a few conjectures and point a few directions for future research. These are particularly informed by my taste.

6.1. Actions of groups with property $(T)$. Lattices in $S P(1, n)$ and $F_{4}^{-20}$ share many of the rigidity properties of higher rank lattices. In light of Theorems 5.1, 5.2 and 5.13, it seems natural to conjecture: 
CONJECTURE 6.1. Let $G$ be a semisimple Lie group with no compact factors and no simple factors isomorphic to $S O(1, n)$ or $S U(1, n)$, and let $\Gamma<G$ be a lattice. Then any volume preserving generalized quasiaffine action of $G$ or $\Gamma$ on a compact manifold is locally rigid.

Note that for $G$ with no rank one factors and quasiaffine actions, this is just Theorem 5.1. To apply the methods of [FM1; FM2; FM3] in the setting of Conjecture 6.1 there are essentially three difficulties:

(1) If the action is generalized quasiaffine and not quasiaffine, then one cannot use the construction of the Margulis-Qian cocycle described above. This is easiest to see for a generalized affine action $\rho$ on some $K \backslash H / \Lambda$. The action $\rho$ lifts to $H / \Lambda$, but a perturbation $\rho^{\prime}$ need not. If $K$ is finite, this difficulty can be overcome by passing to a subgroup of finite index $\Gamma^{\prime}<\Gamma$ and arguments in [FM3] can be used prove rigidity of $\Gamma$ from rigidity of $\Gamma^{\prime}$. If $K$ is compact and connected, this is a genuine and surprisingly intractable difficulty.

(2) Proving a version of Zimmer's cocycle superrigidity for groups as in the assumptions of the conjecture. Partial results in this direction were obtained by Corlette-Zimmer and Korevaar-Schoen, but their results all require hypotheses that are obviously restrictive or simply difficult to verify. Very recently, the author and Hitchman have proven a complete version of cocycle superrigidity, at least for so-called $L^{2}$-cocycles. In light of our work, this difficulty is already overcome.

(3) Replace the method of Katok-Spatzier in the proof that the conjugacy is actually smooth. The proof of Theorem 5.13 gives some progress in this direction, but there are significant technical difficulties to overcome in applying Hitchman's methods at this level of generality. There is some progress on this question by Gorodnik, Hitchman and Spatzier.

The author and Hitchman have another approach to Conjecture 6.1 based on Theorem 5.12, some estimates proven in [FH1], and using heat flow and those estimates to produce a tame splitting of the short exact sequence in Theorem 5.12. It is not yet clear how generally applicable this method will be.

The following question is also interesting in this context:

QUESTION 6.2. Let $G$ and $\Gamma$ be as in Conjecture 6.1. Let $\rho$ be a non-volumepreserving affine action of $\Gamma$ on a compact manifold $M$. When is $\rho$ locally rigid?

By the work of Stuck discussed at the end of Section 4.2, it is clear that local rigidity will not hold in full generality here. In particular for the product of the action of $\Gamma$ on the boundary $G / P$ with the trivial action of $\Gamma$ on any manifold, there is already strong evidence against local rigidity. We give two particularly 
interesting special cases where we expect local rigidity to occur. The first is to take a lattice $\Gamma$ in $S P(1, n)$ and ask if the $\Gamma$ action on the boundary $S P(1, n) / P$ is locally rigid. This is analogous to Theorems 4.16 and 4.18. Another direction worth pursuing is to see if the action of say $S l(n, \mathbb{Z})$ on $\mathbb{R} P^{n+k}$ is locally rigid for $n \geq 3$ and any $k \geq 0$. For cocompact lattices instead of $S L(n, \mathbb{Z})$ and $k=$ -1 it is Theorem 4.16. One can ask a wide variety of similar questions for both compact and noncocompact lattices acting on homogeneous spaces that are "larger than" any natural boundary for the group as long as one avoids settings in which Stuck's examples can occur. We remark that in some instances, partial results for analytic perturbations can be obtained by Zeghib's method [Zg].

We remark here that the action of $S L(n, \mathbb{Z})$ on $\mathbb{T}^{n}$ is not locally rigid in Homeo $\left(\mathbb{T}^{n}\right)$ by a construction of Weinberger. It would be interesting to understand local rigidity in low regularity for other actions.

6.2. Actions of irreducible lattices in products. In this subsection, we formulate a general conjecture concerning local rigidity of actions of irreducible lattices in products. We begin by making a few remarks on other rigidity properties of irreducible lattices and by describing a few examples where rigidity might hold, as well as some examples where it does not.

Rigidity properties of irreducible lattices have traditionally been studied together with rigidity of lattices in simple groups, and irreducible lattices enjoy many of the same rigidity properties. We list a few here to motivate our conjectures on rigidity of actions of these lattices. The properties we list also rule out certain trivial constructions of perturbations and deformations of actions. In the following, $\Gamma$ is an irreducible lattice in $G=\left(\prod_{i=1}^{k} G_{i}\right) / Z$ where each $G_{i}$ is a noncompact semisimple Lie group and $Z$ is a subgroup of the center of $\prod_{i=1}^{k} G_{i}$. Many of these results hold more generally, see below.

Properties of irreducible lattices:

(1) There are no nontrivial homomorphisms $\Gamma \rightarrow \mathbb{Z}$ (and therefore no nontrivial homomorphisms to any abelian or nonabelian free group).

(2) All linear representations of $\Gamma$ are classified. In particular, given any representation $\rho$ of $\Gamma$ into $G L(V)$, where $V$ is a finite dimensional vector space, then $H^{1}(\Gamma, V)=0$.

(3) All normal subgroups of $\Gamma$ are either finite or finite index.

The first property, for $\Gamma$ cocompact, was originally proven by Bernstein and Kazhdan [BK]. Some special cases of this result were proven earlier by Matsushima and Shimura [MS]. All other properties are originally due to Margulis, see [Ma4] and historical references there. The original proofs all go through with only minor adaptations if some of the $G_{i}$ are replaced with the $k$-points of a $k$-algebraic group over some other local field $k$. These properties have been 
shown to hold for appropriate classes of lattices in even more general products of locally compact groups by Bader, Monod and Shalom, [Sm; Md1; Md2; BSh].

Example 1: Let $\Gamma=S L(2, \mathbb{Z}[\sqrt{2}])$. We embed $\Gamma$ in $S L(2, \mathbb{R}) \times S L(2, \mathbb{R})$ by taking $\gamma$ to $(\gamma, \sigma(\gamma))$ where $\sigma$ is the Galois automorphism of $\mathbb{Q}(\sqrt{2})$ taking $\sqrt{2}$ to $-\sqrt{2}$. This embedding defines an action of $\Gamma$ on $\mathbb{T}^{4}$ where $\mathbb{T}^{4}=\mathbb{R}^{4} / \mathbb{Z}^{4}$ where we identity $\mathbb{Z}^{4}$ with the image in $\mathbb{R}^{4}$ of $\mathbb{Z}[\sqrt{2}]^{2}$ via the embedding $v \rightarrow(v, \sigma(v))$. We first note that the list of properties given above imply one can prove deformation rigidity of this action using Hurder's argument from [H1] to produce a continuous conjugacy and using the method of Katok-Spatzier [KS1; KS2] to show that the conjugacy is smooth. Anatole Katok has suggested one might be able to show local rigidity of this action by using the methods in [KL1]. This example is just the first in a large class of Anosov actions of irreducible lattices, all of which should be locally rigid. We leave the general construction to the interested reader.

Example 2: We take the action of $\Gamma=S L\left(2, \mathbb{Z}[\sqrt{2}]\right.$ and let $\Gamma$ act on $\mathbb{T}^{5}=$ $\mathbb{T}^{4} \times \mathbb{T}^{1}$ by a diagonal action where the action on $\mathbb{T}^{4}$ is as defined in Example 1 and the action on $\mathbb{T}^{1}$ is trivial. Once again, this is merely the first example of a large class of partially hyperbolic actions of irreducible lattices. In this instance, the central foliation for the $\Gamma$ action consists of compact tori. For this type of example, many of the argument of [NT1; NT2; T] carry over, but the fact that $\Gamma$ does not have property $(T)$ prevents one from using those outlines to prove local rigidity. On the other hand, the methods of [NT1] can be adapted to prove deformation rigidity again replacing their argument for smoothness of the conjugacy by the Katok-Spatzier method. We remark that it is also possible to give many examples of actions of irreducible lattices in products where the central foliation is by dense leaves, and the methods of Nitica and Torok cannot be applied. See discussion below for the obstructions to applying the methods of [FM1; FM2; FM3].

Example 3: We now give an example of a family of actions which extend to an action of $G=G_{1} \times G_{2}$. Let $H$ be a simple Lie group with $G<H$, for example, $H=S L(4, \mathbb{R})$ or $H=S p(4, \mathbb{R})$ and $\Lambda<H$ a cocompact lattice. Then both $G$ and $\Gamma$ act by left translations on $H / \Lambda$.

Example 4:We end with a family of examples for which there exists a large family of deformations. Let $\Gamma$ be as above and let $\Lambda<S L(2, \mathbb{R})=G_{1}$ be an irreducible lattice. Then $\Gamma$ acts by left translations on $M=S L(2, \mathbb{R}) / \Lambda$. Call this action $\bar{\rho}$. I do not currently know whether it is possible to deform this action, but one can use this action to build actions with perturbations on a slightly larger manifold. Let $\Gamma$ act trivially on any manifold $N$ and take the diagonal action $\rho$ on $M \times N$. It is well-known that there exists a nontrivial homomorphism $\sigma: \Lambda \rightarrow \mathbb{Z}$. There is also a standard construction of a cocycle $\alpha: G_{1} \times G_{1} / \Lambda \rightarrow \Lambda$ 
over the $G_{1}$ action on $G_{1} / \Lambda$. The cocycle is defined by taking a fundamental domain $X$ for $\Lambda$ in $G_{1}$, identifying $G_{1} / \Lambda$ with $X$ and letting $\alpha(g, x)$ be the element of $\Lambda$ such that $g x \alpha(g, x)^{-1}$, as an element of $G_{1}$, is in $X$. Taking any vector field $V$ on $N$ and let $s_{\varepsilon}$ be the $\mathbb{Z}$ action on $N$ defined by having 1 act by flowing to time $\varepsilon$ along $V$. We then define a $\Gamma$ action on $M \times N$ by taking

$$
\rho_{\varepsilon}(\gamma)(m, n)=\left(\bar{\rho}(\gamma) m, s_{\varepsilon}(\sigma(\alpha(g, m))(n)) .\right.
$$

We leave it to the interested reader to show that the actions $\rho_{\varepsilon}$ are not conjugate to $\rho$ for essentially any choice of $V$.

The key point in example 4, which is not present in the first three examples, is that the action factors through a projection of $\Gamma$ into a simple factor of $G$. Motivated by the examples so far, by the results in Theorem 5.10, and by analogy with results on actions of higher rank abelian groups, we make the following definition and conjecture:

DEFINITION 6.3. Let $G=G_{1} \times \cdots \times G_{k}$ be a semisimple Lie group where all the $G_{i}$ are noncompact. Let $\Gamma<G$ be an irreducible lattice. Let $\rho$ be an affine action of $\Gamma$ on some $H / \Lambda$ where $H$ is a Lie group and $\Lambda<H$ is a cocompact lattice. Then we say $\rho$ has rank one factors if there exists

(1) an action $\bar{\rho}$ of $\Gamma$ on a some space $X$ which is a factor of $\rho$

(2) and a rank one factor $G_{i}$ of $G$

such that $\bar{\rho}$ is the restriction of a $G_{i}$ action. I.e. $\Gamma$ acts on $X$ by projecting $\Gamma$ to $G_{i}$ and restricting a $G_{i}$ action.

Conjecture 6.4. Let $G, \Gamma, H, \Lambda$ and $\rho$ be as in Definition 6.3. Then if $\rho$ has no rank one factors $\rho$ is locally rigid.

REMARK. It is a consequence of Ratner's measure rigidity theorem, see [R; $\mathrm{Sh}$; W2], that any rank one factor of an affine action for these groups is in fact affine. This implies that any rank one factor is a left translation action on some $H^{\prime} / \Lambda^{\prime}$. So a special case of the conjecture is that any affine $\Gamma$ action on a torus or nilmanifold is locally rigid.

There is a variant of Conjecture 6.4 for $G$ actions. We recall that a measure preserving action of $G=G_{1} \times \cdots \times G_{k}$ action is irreducible if each $G_{i}$ acts ergodically. We extend this notion to nonergodic $G$ actions by saying that the action is weakly irreducible if every ergodic component of the volume measure for the action of any $G_{i}$ is an ergodic component of the volume measure for the action of $G$.

Conjecture 6.5. Let $G, H, \Lambda$ be as in Definition 6.3 and let $G<H$, so that we have a left translation action $\rho$ of $G$ on $H / \Lambda$. Then if $\rho$ is weakly irreducible, $\rho$ is locally rigid. 
The relation of the two conjectures follows from the following easy lemma.

Lemma 6.6. Let $G, \Gamma, H, \Lambda$ and $\rho$ be as in Definition 6.3, then the $\Gamma$ action on $H / \Lambda$ has no rank one factors if and only if the induced $G$ action on $(G \times H / \Lambda) / \Gamma$ is weakly irreducible.

To prove the lemma requires both some algebraic untangling of induced actions and a use of Margulis' superrigidity theorem to describe affine actions of $G$ and $\Gamma$ along the lines of [FM1, Theorems 6.4 and 6.5]. We leave this as an exercise for the interested reader. It is fairly easy to check the lemma for any particular affine $\Gamma$ action.

We end this subsection by pointing out the difficulty in approaching this conjecture by means of the methods of [FM1; FM2; FM3]. A central difficulty is that the lattices in question do not have property $(T)$ and so the foliated generalization of Theorem 5.2 does not apply. However, even in the case of weakly hyperbolic actions, there are significant difficulties. To begin the argument, one would like to apply cocycle superrigidity to the Margulis-Qian cocycle. To do this requires the existence of an invariant measure which is usually established using property $(T)$ by an argument of Seydoux [Sy]. In this setting, where property $(T)$ does not hold, one might try instead to use the work of Nevo and Zimmer, [NZ1; NZ2; NZ3], but there are nontrivial difficulties here as well. One cannot apply their theorems without first showing that the perturbed action satisfies some irreducibility assumption. Even if one were to obtain an invariant measure, the precise form of cocycle superrigidity required is not known for products of rank one groups or their irreducible lattices. And the strongest possible forms of cocycle superrigidity in this context again require a kind of irreducibility of the perturbed action. So to proceed by this method one would need to show that perturbations of the actions in Conjecture 6.4 and 6.5 still satisfied some irreducibility conditions. This seems quite difficult. It may also be possible to approach these questions by using Theorem 5.12, but even proving that the relevant cohomology groups vanish seems subtle.

6.3. Other questions and conjectures. We end this article by discussing some other questions and conjectures.

In the context of Theorem 5.11 it is interesting to ask if isometric actions of lattices in $S U(1, n)$ are locally rigid. For some choices of lattice, the answer is trivially no. Namely some cocompact lattices in $S U(1, n)$ have homomorphisms $\rho$ to $\mathbb{Z}[\mathrm{Ka} 2$; BW], and so have arithmetic actions with deformations provided the centralizer $Z$ of $K$ in $\operatorname{Diff}^{\infty}(M)$ is nontrivial. Having centralizer allows one to deform the action along the image of the homomorphism $\rho \circ \sigma_{t}: F \rightarrow Z$ where $\sigma_{t}: \mathbb{Z} \rightarrow Z$ is any one parameter family of homomorphisms. It seems reasonable to conjecture: 
CONJECTURE 6.7. Let $\rho$ be an arithmetic isometric action of a lattice in $S U(1, n)$. Then any sufficiently small perturbation of $\rho$ is of the form described in the previous paragraph.

This conjecture is in a certain sense an infinite dimensional analogue of work of Goldman-Millson and Corlette [Co1; GM]. Another conjecture concerning complex hyperbolic lattices, for which work of Yue provides significant evidence [Yu], is:

CONJECTURE 6.8. Is the action of any lattice in $S U(1, n)$ on the boundary of complex hyperbolic space locally rigid?

There are also many interesting questions concerning the failure of local rigidity for lattices in $S O(1, n)$. The only rigidity theorem we know of in this context is Kanai's, Theorem 4.17, and it would be interesting to extend Kanai's theorem to nonuniform lattices. In [F1; F3] various deformations of lattices in $S O(1, n)$ are constructing for affine and isometric actions. These constructions both adapt the bending construction of Johnson and Millson, [JM]. It seems likely that in some cases one should be able to prove results concerning the structure of the representation space and, in particular, to show that it is "singular" in an appropriate sense. See [F3] for more discussion.

Two other paradigmatic examples of large groups are the outer automorphism group of the free group, $\operatorname{Out}\left(F_{n}\right)$, and the mapping class group of a surface $S, M C G(S)$. These groups do not admit many natural actions on compact manifolds, but there are some natural interesting actions quite analogous to those we have already discussed. For $M C G(S)$, the question we raise here is already raised in $[\mathrm{La}]$. The actions we consider are "nonlinear" analogues of the standard actions of $S L(n, \mathbb{Z})$ on $\mathbb{T}^{n}$ and $S P(2 n, \mathbb{Z})$ on $\mathbb{T}^{2 n}$. The spaces acted upon are moduli spaces of representations of either the free group or the fundamental group of a surface $S$, where the representations take values in compact groups. More precisely, we have an action of $\operatorname{Out}\left(F_{n}\right)$ on $\operatorname{Hom}\left(F_{n}, K\right) / K$ and an action of $M C G(S)$ on $\operatorname{Hom}(\pi(S), K) / K$ where $K$ is a compact group. It is natural to ask whether these actions are locally rigid, though the meaning of the question is somewhat obscured by the fact that the representation varieties are not smooth. For $K=S^{1}$, one obtains actions on manifolds, and in fact tori, and one might begin by considering that case.

We end with a question motivated by the recent work of Damjanovic and Katok. We only give a special case here. Let $G$ be a real split, simple Lie group of real rank at least two. Let $\Gamma<G \times G$ be an irreducible lattice. Let $K<G$ be a maximal compact subgroup and view $K$ as a subgroup of $G \times G$ by viewing it as a subgroup of the second factor. The quotient $K \backslash(G \times G) / \Gamma$ has a natural $G$ 
action on the left on the first factor. We can restrict this action to the action of a maximal split torus $A$ in $G$. Note that $A$ is isomorphic to $\mathbb{R}^{d}$ for some $d \geq 2$.

QUESTION 6.9. Is the action of $\mathbb{R}^{d}$ described in the paragraph above locally rigid?

\section{References}

[An] Anosov, D. V. Geodesic flows on closed Riemann manifolds with negative curvature. Proceedings of the Steklov Institute of Mathematics, No. 90 (1967). Translated from the Russian by S. Feder. American Mathematical Society, Providence, RI, 1969.

[AN] An, J.; Neeb, K. An implicit function theorem for Banach spaces and some applications. Preprint.

[BSh] Bader, U.; Shalom, Y. Factor and normal subgroup theorems for lattices in products of groups. Preprint.

[Ba1] Banyaga, A. Sur la structure du groupe des difféomorphismes qui préservent une forme symplectique. Comment. Math. Helv. 53 (1978), No. 2, 174-227.

[Ba2] Banyaga, A. The structure of classical diffeomorphism groups. Mathematics and its Applications, No. 400. Kluwer, Dordrecht, 1997.

[Be1] Benveniste, E. J. Rigidity of isometric lattice actions on compact Riemannian manifolds. GAFA 10 (2000), 516-542.

[Be2] Benveniste, E. J. Exotic actions of semisimple groups and their deformations. Unpublished preprint and Chapter 2 of University of Chicago Ph.D. dissertation, 1996.

[BF] Benveniste, E. J.; Fisher, D. Non-existence of invariant rigid structures and invariant almost rigid structures. To appear in Comm. Anal. Geom., 21 pages. http:// comet.lehman.cuny.edu/fisher/

[BK] Bernstein, I. N.; Kazhdan, D. A. The one-dimensional cohomology of discrete subgroups. (Russian). Funkcional. Anal. i Priložen. 4 (1970), No. 1, 1-5.

[BCG] Besson, G.; Courtois, G.; Gallot, S. Minimal entropy and Mostow's rigidity theorems. Ergodic Theory Dynam. Systems 16 (1996), No. 4, 623-649.

[Bo] Borel, A. Compact Clifford-Klein forms of symmetric spaces. Topology 2 (1963), 111-122.

[BW] Borel, A.; Wallach, N. Continuous cohomology, discrete subgroups, and representations of reductive groups. Second edition. Math. Surveys Monogr., No. 67. American Mathematical Society, Providence, RI, 2000.

[BuW] Burslem, E.; Wilkinson, A. global rigidity of solvable group actions on $S^{1}$. Geom. Topol. 8 (2004), 877-924.

[BPSW] Burns, Keith; Pugh, Charles; Shub, Michael; Wilkinson, Amie. Recent results about stable ergodicity. Smooth ergodic theory and its applications (Seattle, WA, 1999), 327-366. Proc. Sympos. Pure Math., No. 69. American Mathematical Society, Providence, RI, 2001. 
[CGh] Cairns, Grant; Ghys, Étienne. The local linearization problem for smooth SL(n)actions. Enseign. Math. (2) 43 (1997), No. 1-2, 133-171.

[C] Calabi, E. On compact, Riemannian manifolds with constant curvature, I. Proc. Sympos. Pure Math., No. 3, 155-180. American Mathematical Society, Providence, RI, 1961.

[CV] Calabi, E.; Vesentini, E. On compact, locally symmetric Kähler manifolds. Ann. of Math. (2) 71 (1960), 472-507.

[Cl] Clozel, Laurent. On the cohomology of Kottwitz's arithmetic varieties. Duke Math. J. 72 (1993), No. 3, 757-795.

[CF] Connell, Christopher; Farb, Benson. Some recent applications of the barycenter method in geometry. Topology and geometry of manifolds (Athens, GA, 2001), 1950. Proc. Sympos. Pure Math., No. 71. American Mathematical Society, Providence, RI, 2003.

[Co1] Corlette, Kevin. Flat G-bundles with canonical metrics. J. Differential Geom. 28 (1988), No. 3, 361-382.

[Co2] Corlette, Kevin. Archimedean superrigidity and hyperbolic geometry. Ann. of Math. (2) 135 (1992), No. 1, 165-182.

[DK1] Damjanovich, D.; Katok, A. Local rigidity of partially hyperbolic actions of $\mathbb{Z}^{k}$ and $\mathbb{R}^{k}, k \geq 2$, I. KAM method and actions on the torus. Preprint. http:// www.math.psu.edu/katok_a/papers.html

[DK2] Damjanović, Danijela; Katok, Anatole. Local rigidity of actions of higher rank abelian groups and KAM method. Electron. Res. Announc. Amer. Math. Soc. 10 (2004), 142-154.

[DK3] Damjanović, Danijela; Katok, Anatole. Periodic cycle functions and cocycle rigidity for certain partially hyperbolic $\mathbb{R}^{k}$ actions. Discrete Contin. Dynam. Systems 13 (2005), No. 4, 985-1005.

[dlLMM] de la Llave, R.; Marco, J. M.; Moriyón, R. Canonical perturbation theory of Anosov systems and regularity results for the Livšic cohomology equation. Ann. of Math. (2) 123 (1986), No. 3, 537-611.

[De] Delorme, Patrick. 1-cohomologie des représentations unitaires des groupes de Lie semi-simples et résolubles. Produits tensoriels continus de représentations. Bull. Soc. Math. France 105 (1977), No. 3, 281-336.

[EF] Einsiedler, M.; Fisher, T. Differentiable rigidity for hyperbolic toral actions. Preprint.

[Ep] Epstein, D. B. A. The simplicity of certain groups of homeomorphisms. Compositio Math. 22 (1970), 165-173.

[Fe1] Feres, Renato. Dynamical systems and semisimple groups: an introduction. Cambridge Tracts in Math., No. 126. Cambridge University Press, Cambridge, 1998.

[Fe2] Feres, Renato. An introduction to cocycle super-rigidity. Rigidity in dynamics and geometry (Cambridge, 2000), 99-134. Springer, Berlin, 2002. 
[FK] R. Feres; Katok, A. Ergodic theory and dynamics of G-spaces (with special emphasis on rigidity phenomena). Handbook of dynamical systems, $1 \mathrm{~A}$, edited by $\mathrm{B}$. Hasselblatt and A. Katok, 665-763. Elsevier, Amsterdam, 2002. http://www.math. wustl.edu/feres/publications.html.

[F1] Fisher, D. Deformations of group actions. Preprint, 14 pages, to appear in Transactions AMS. http://comet.lehman.cuny.edu/fisher/

[F2] Fisher, D. First cohomology and local rigidity of group actions. In preparation.

[F3] Fisher, D. Bending group actions and cohomology of arithmetic lattices in $S O(1, n)$. In preparation.

[FH1] Fisher, D.; Hitchman, T. J. Strengthening property $(T)$ by Bochner methods. Preprint.

[FH2] Fisher, D.; Hitchman, T. J. Superrigidity for cocycles via Bochner methods. In preparation.

[FM1] Fisher, D.; Margulis, G. A. Local rigidity for cocycles. Papers in honor of Calabi, Lawson, Siu and Uhlenbeck, 191-234. Surv. Diff. Geom., No. VIII. S.-T. Yau (ed.). International Press, Somerville, MA, 2003.

[FM2] Fisher, D.; Margulis, G. A. Almost isometries, property $(T)$ and local rigidity. To appear in Invent. Math.

[FM3] Fisher, D.; Margulis, G. A. Local rigidity for affine actions of higher rank Lie groups and their lattices. Preprint, 59 pages. http://comet.lehman.cuny.edu/fisher/

[FW] Fisher, D.; Whyte, K. Continuous quotients for lattice actions on compact spaces. Geom. Dedicata 87 (2001), 181-189.

[Fl] Fleming, Philip J. Structural stability and group cohomology. Trans. Amer. Math. Soc. 275 (1983), No. 2, 791-809.

[Fu1] Furstenberg, Harry. A Poisson formula for semi-simple Lie groups. Ann. of Math. (2) 77 (1963), 335-386.

[Fu2] Furstenberg, Harry. Boundaries of Lie groups and discrete subgroups. Actes du Congrès International des Mathématiciens (Nice, 1970), Tome 2, 301-306. Gauthier-Villars, Paris, 1971.

[Fu3] Furstenberg, Harry. Rigidity and cocycles for ergodic actions of semisimple Lie groups (after G. A. Margulis and R. Zimmer). Bourbaki Seminar, 1979/80, 273-292. Lecture Notes in Math., No. 842. Springer, Berlin, 1981.

[GhS] Ghys, E.; Sergiescu, V. Stabilité et conjugaison différentiable pour certains feuilletages. Topology 19 (1980), No. 2, 179-197.

[Gh1] Ghys, Étienne. Actions localement libres du groupe affine. [Locally free actions of the affine group]. Invent. Math. 82 (1985), No. 3, 479-526.

[Gh2] Ghys, Étienne. Déformations de flots d'Anosov et de groupes fuchsiens. [Deformations of Anosov flows and Fuchsian groups]. Ann. Inst. Fourier (Grenoble) $\mathbf{4 2}$ (1992), No. 1-2, 209-247. 
[Gh3] Ghys, Étienne. Rigidité différentiable des groupes fuchsiens. [Differentiable rigidity of Fuchsian groups]. Publ. Math. IHES 78 (1993), 163-185.

[GM] Goldman, W. M.; Millson, J. J. Local rigidity of discrete groups acting on complex hyperbolic space. Invent. Math. 88 (1987), No. 3, 495-520.

[GS] Gromov, Mikhail; Schoen, Richard. Harmonic maps into singular spaces and $p$ adic superrigidity for lattices in groups of rank one. Publ. Math. IHES 76 (1992), $165-246$.

[Gu] Guichardet, Alain. Sur la cohomologie des groupes topologiques, II. Bull. Sci. Math. (2) 96 (1972), 305-332.

[G] Guysinsky, M. The theory of nonstationary normal forms. Ergodic Theory Dynam. Systems 22 (2002), No. 3, 845-862.

[GK] Guysinsky, M.; Katok, A. Normal forms and invariant geometric structures on transverse contracting foliations. Math. Res. Lett. 5 (1998), 149-163.

[HV] de la Harpe, P.; Valette, A. La propriete (T) de Kazhdan pour les groupes localement compacts. Asterisque, No. 175. Soc. Math. de France, Paris, 1989.

[Ha1] Hamilton, Richard S. The inverse function theorem of Nash and Moser. Bull. Amer. Math. Soc. 7 (1982), No. 1, 65-222.

[Ha2] Hamilton, Richard S. Deformation of complex structures on manifolds with boundary, I: The stable case. J. Differential Geom. 12 (1977), No. 1, 1-45.

[He] Helgason, Sigurdur. Differential geometry, Lie groups, and symmetric spaces. Corrected reprint of the 1978 original. Grad. Stud. Math., No. 34. American Mathematical Society, Providence, RI, 2001.

[Hr] Herman, Michael-Robert. Simplicité du groupe des difféomorphismes de classe $C^{\infty}$, isotopes à l'identité, du tore de dimension n. C. R. Acad. Sci. Paris Sér. A-B 273 (1971), A232-A234.

[HPS] Hirsch, M. W.; Pugh, C. C.; Shub, M. Invariant Manifolds. Lecture Notes in Math., No. 583. Springer, New York, 1977.

[Hi] Hitchman, T. J. Rigidity theorems for large dynamical systems with hyperbolic behavior. Ph. D. Thesis, University of Michigan, 2003.

[H1] Hurder, Steven. Rigidity for Anosov actions of higher rank lattices. Ann. of Math. (2) 135 (1992), No. 2, 361-410.

[H2] Hurder, Steven. Infinitesimal rigidity for hyperbolic actions. J. Differential Geom. 41 (1995), No. 3, 515-527.

[JM] Johnson, Dennis; Millson, John J. Deformation spaces associated to compact hyperbolic manifolds. Discrete groups in geometry and analysis (New Haven, CT, 1984), 48-106. Progr. Math., No. 67. Birkhäuser, Boston, 1987.

[Jn] Journé, J.-L. A regularity lemma for functions of several variables. Rev. Mat. Iberoamericana 4 (1988), No. 2, 187-193.

[Kan1] Kanai, M. A new approach to the rigidity of discrete group actions. Geom. Funct. Anal. 6 (1996), No. 6, 943-1056. 
[Kan2] Kanai, Masahiko. A remark on local rigidity of conformal actions on the sphere. Math. Res. Lett. 6 (1999), No. 5-6, 675-680.

[KL1] Katok, A.; Lewis, J. Local rigidity for certain groups of toral automorphisms. Israel J. Math. 75 (1991), No. 2-3, 203-241.

[KL2] Katok, A.; Lewis, J. Global rigidity results for lattice actions on tori and new examples of volume-preserving actions. Israel J. Math. 93 (1996), 253-280.

[KLZ] Katok, A.; Lewis, J.; Zimmer, R. Cocycle superrigidity and rigidity for lattice actions on tori. Topology 35 (1996), No. 1, 27-38.

[KNT] Katok, Anatole; Niţică, Viorel; Török, Andrei. Non-abelian cohomology of abelian Anosov actions. Ergodic Theory Dynam. Systems 20 (2000), No. 1, 259288.

[KS1] Katok, A.; Spatzier, R. J. Nonstationary normal forms and rigidity of group actions. Electron. Res. Announc. Amer. Math. Soc. 2 (1996), No. 3, 124-133.

[KS2] Katok, A.; Spatzier, R. J. Differential rigidity of Anosov actions of higher rank abelian groups and algebraic lattice actions. Din. Sist. i Smezhnye Vopr., 292-319. Tr. Mat. Inst. Steklova, No. 216 (1997). Translation in Proc. Steklov Inst. Math. 216 (1997), No. 1, 287-314.

[KS3] Katok, Anatole; Spatzier, Ralf J. First cohomology of Anosov actions of higher rank abelian groups and applications to rigidity. Publ. Math. IHES 79 (1994), 131156.

[KS4] Katok, A.; Spatzier, R. J. Subelliptic estimates of polynomial differential operators and applications to rigidity of abelian actions. Math. Res. Lett. 1 (1994), No. 2, 193-202.

[Ka1] Kazhdan, D. A. On the connection of the dual space of a group with the structure of its closed subgroups. (Russian). Funkcional. Anal. i Priložen. 1 (1967), 71-74.

[Ka2] Kazhdan, David. Some applications of the Weil representation. J. Analyse Mat. 32 (1977), 235-248.

[Kn] Knapp, Anthony W. Lie groups beyond an introduction. Second edition. Prog. Math., No. 140. Birkhäuser, Boston, 2002.

[Ko] Kononenko, A. Infinitesimal rigidity of boundary lattice actions. Ergodic Theory Dynam. Systems 19 (1999), No. 1, 35-60.

[KY] Kononenko, A.; Yue, C. B. Cohomology and rigidity of Fuchsian groups. Israel J. Math. 97 (1997), 51-59.

[La] Labourie, François. Large groups actions on manifolds. Proceedings of the International Congress of Mathematicians, II (Berlin, 1998). Doc. Math. Extra Vol. II (1998), 371-380 (electronic).

[L] Lewis, J. Infinitesimal rigidity for the action of $S L_{n}(\mathbb{Z})$ on $\mathbb{\mathbb { V }}^{n}$. Trans. Amer. Math. Soc. 324 (1991), No. 1, 421-445.

[LZ] Lubotzky, Alexander; Zimmer, Robert J. Variants of Kazhdan's property for subgroups of semisimple groups. Israel J. Math. 66 (1989), No. 1-3, 289-299. 
[Ma1] Margulis, G. A. On the action of unipotent groups in the space of lattices. Lie groups and their representations (Budapest, 1971), 365-370. Halsted, New York, 1975.

[Ma2] Margulis, G. A. Arithmetic properties of discrete subgroups. (Russian). Uspehi Mat. Nauk 29 (1974), No. 1 (175), 49-98.

[Ma2] Margulis, G. A. Discrete groups of motions of manifolds of non-positive curvature. Proc. Int. Cong. Math. (Vancouver, 1974). Amer. Math. Soc. Transl. 109 (1977), 33-45.

[Ma3] Margulis, G. A. Arithmeticity of irreducible lattices in the semisimple groups of rank greater than 1. Appendix to the Russian translation of M. Ragunathan, Discrete subgroups of Lie groups, Mir, Moscow, 1977 (in Russian). English translation in Invent. Math. 76 (1984), 93-120.

[Ma4] Margulis, G. A. Discrete subgroups of semisimple Lie groups. Springer, New York, 1991.

[MQ] Margulis, G.; Qian, N. Local rigidity of weakly hyperbolic actions of higher real rank semisimple Lie groups and their lattices. Ergodic Theory Dynam. Systems $\mathbf{2 1}$ (2001), 121-164.

[Mt1] Mather, John N. Integrability in codimension 1. Comment. Math. Helv. 48 (1973), 195-233.

[Mt2] Mather, John N. Commutators of diffeomorphisms. Comment. Math. Helv. 49 (1974), 512-528.

[Mt3] Mather, John N. Foliations and local homology of groups of diffeomorphisms. Proceedings of the International Congress of Mathematicians (Vancouver, BC, 1974), Vol. 2, 35-37. Canadian Mathematical Congress, Montreal, 1975.

[MM] Matsushima, Yozō; Murakami, Shingo. On vector bundle valued harmonic forms and automorphic forms on symmetric riemannian manifolds. Ann. of Math. (2) 78 (1963), 365-416.

[MS] Matsushima, Yozō; Shimura, Goro. On the cohomology groups attached to certain vector valued differential forms on the product of the upper half planes. Ann. of Math. (2) 78 (1963), 417-449.

[Md1] Monod, Nicolas. Superrigidity for irreducible lattices and geometric splitting. Preprint.

[Md2] Monod, Nicolas. Arithmeticity vs. non-linearity for irreducible lattices. To appear in Geom. Dedicata.

[Mr] Morris, Dave Witte. An introduction to arithmetic groups. Book in preprint form.

[Mo1] Mostow, G. D. Strong rigidity of locally symmetric spaces. Ann. of Math. Stud., No. 78. Princeton University Press, Princeton, NJ, 1973.

[Mo2] Mostow, G. D. Personal communication.

$[\mathrm{Mu}]$ Mumford, D.; Fogarty, J.; Kirwan, F. Geometric invariant theory. Third edition. Ergeb. Math. Grenzgeb. (3), No. 34. Springer, Berlin, 1994. 
[NZ1] Nevo, Amos; Zimmer, Robert J. Homogenous projective factors for actions of semi-simple Lie groups. Invent. Math. 138 (1999), No. 2, 229-252.

[NZ2] Nevo, Amos; Zimmer, Robert J. Rigidity of Furstenberg entropy for semisimple Lie group actions. Ann. Sci. École Norm. Sup. (4) 33 (2000), No. 3, 321-343.

[NZ3] Nevo, Amos; Zimmer, Robert J. A structure theorem for actions of semisimple Lie groups. Ann. of Math. (2) 156 (2002), No. 2, 565-594.

[NT1] Nitica, Viorel; Török, Andrei. Cohomology of dynamical systems and rigidity of partially hyperbolic actions of higher-rank lattices. Duke Math. J. 79 (1995), No. $3,751-810$.

[NT2] Nitica, Viorel; Török, Andrei. Local rigidity of certain partially hyperbolic actions of product type. Ergodic Theory Dynam. Systems 21 (2001), No. 4, 12131237.

[Q1] Qian, Nan Tian. Topological deformation rigidity of higher rank lattice actions. Math. Res. Lett. 1 (1994), No. 4, 485-499.

[Q2] Qian, Nantian. Anosov automorphisms for nilmanifolds and rigidity of group actions. Ergodic Theory Dynam. Systems 15 (1995), No. 2, 341-359.

[Q3] Qian, Nantian. Infinitesimal rigidity of higher rank lattice actions. Comm. Anal. Geom. 4 (1996), No. 3, 495-524.

[QY] Qian, Nantian; Yue, Chengbo. Local rigidity of Anosov higher-rank lattice actions. Ergodic Theory Dynam. Systems 18 (1998), No. 3, 687-702.

[Rg1] Raghunathan, M. S. On the first cohomology of discrete subgroups of semisimple Lie groups. Amer. J. Math. 87 (1965), 103-139.

[R] Ratner, M. On Raghunathan's measure conjectures. Ann. of Math. (2) 134 (1991), No. 3, 545-607.

[S] Selberg, A. On discontinuous groups in higher-dimensional symmetric spaces. Contributions to function theory (Bombay, 1960), 147-164. Internat. Colloq. Function Theory. Tata Institute of Fundamental Research, Bombay, 1960.

[Sy] Seydoux, G. Rigidity of ergodic volume-preserving actions of semisimple groups of higher rank on compact manifolds. Trans. Amer. Math. Soc. 345 (1994), 753-776.

[Sh] Shah, N. Invariant measures and orbit closures on homogeneous spaces for actions of subgroups generated by unipotent elements. Lie groups and ergodic theory (Mumbai, 1996), 229-271. Tata Inst. Fund. Res. Stud. Math., No. 14. S. G. Dani (ed.). Tata Institute of Fundamental Research, Bombay, 1998.

[S11] Shalom, Yehuda. Rigidity of commensurators and irreducible lattices. Invent. Math. 141 (2000), No. 1, 1-54.

[S12] Shalom, Yehuda. Measurable group theory. Preprint.

[Sm] Smale, S. Differentiable dynamical systems. Bull. Amer. Math. Soc. 73 (1967), 747-817. 
[Sp1] Spatzier, R. J. Harmonic analysis in rigidity theory. Ergodic theory and its connections with harmonic analysis (Alexandria, 1993), 153-205. London Math. Soc. Lecture Note Ser., No. 205. Cambridge University Press, Cambridge, 1995.

[Sp2] Spatzier, R. J. An invitation to rigidity theory. Modern dynamical systems and applications, 211-231. Cambridge University Press, Cambridge, 2004.

[St1] Stowe, Dennis. The stationary set of a group action. Proc. Amer. Math. Soc. 79 (1980), No. 1, 139-146.

[St2] Stowe, Dennis. Stable orbits of differentiable group actions. Trans. Amer. Math. Soc. 277 (1983), No. 2, 665-684.

[Sk] Stuck, Garrett. Minimal actions of semisimple groups. Ergodic Theory Dynam. Systems 16 (1996), No. 4, 821-831.

[Th1] Thurston, W. A generalization of the Reeb stability theorem. Topology 13 (1974), 347-352.

[Th2] Thurston, William. Foliations and groups of diffeomorphisms. Bull. Amer. Math. Soc. 80 (1974), 304-307.

[T] Török, Andrei. Rigidity of partially hyperbolic actions of property (T) groups. Discrete Contin. Dynam. Systems 9 (2003), No. 1, 193-208.

[We1] Weil, André. On discrete subgroups of Lie groups. Ann. of Math. (2) 72 (1960), 369-384.

[We2] Weil, André. On discrete subgroups of Lie groups, II. Ann. of Math. (2) 75 (1962), 578-602.

[We3] Weil, André. Remarks on the cohomology of groups. Ann. of Math. (2) 80 (1964), 149-157.

[W1] Witte, Dave. Cocompact subgroups of semisimple Lie groups. Lie algebra and related topics (Madison, WI, 1988), 309-313. Contemp. Math., No. 110. American Mathematical Society, Providence, RI, 1990.

[W2] Witte, D. Measurable quotients of unipotent translations on homogeneous spaces. Trans. Amer. Math. Soc. 354 (1994), No. 2, 577-594.

[Yk] Yaskolko, S. Penn State Ph. D. dissertation, 1999.

[Yu] Yue, Cheng Bo. Smooth rigidity of rank-1 lattice actions on the sphere at infinity. Math. Res. Lett. 2 (1995), No. 3, 327-338.

[Zg] Zeghib, Abdelghani. Quelques remarques sur les actions analytiques des réseaux des groupes de Lie de rang supérieur. [Some remarks on analytic actions of lattices of Lie groups of higher rank]. C. R. Acad. Sci. Paris Sér. I Math. 328 (1999), No. 9, 799-804.

[Z1] Zimmer, R. J. Volume preserving actions of lattices in semisimple groups on compact manifolds. Publ. Math. IHES 59 (1984), 5-33.

[Z2] Zimmer, R. J. Ergodic theory and semisimple groups. Birkhäuser, Boston, 1984.

[Z3] Zimmer, R. J. Lattices in semisimple groups and distal geometric structures. Invent. Math. 80 (1985), 123-137. 
[Z4] Zimmer, R. J. Actions of semisimple groups and discrete subgroups. Proc. Internat. Cong. Math. (Berkeley, 1986), 1247-1258.

[Z5] Zimmer, R. J. Lattices in semisimple groups and invariant geometric structures on compact manifolds. Discrete groups in geometry and analysis: Papers in honor of G. D. Mostow (New Haven, CT, 1984), 152-210. Roger Howe (ed.). Progr. Math., No. 67. Birkhäuser, Boston, 1987.

[Z6] Zimmer, Robert J. Infinitesimal rigidity for smooth actions of discrete subgroups of Lie groups. J. Differential Geom. 31 (1990), No. 2, 301-322.

\section{DAVID FISHER}

DEPARTMENT OF MATHEMATICS

RAWLES HALL

INDIANA UNIVERSITY

BLOOMINGTON, IN 47405

fisherdm@indiana.edu 
